\title{
Wake of inertial waves of a horizontal cylinder in horizontal translation
}

\author{
Nathanaël Machicoane, ${ }^{1}$ Vincent Labarre, ${ }^{1}$ Bruno Voisin, ${ }^{2}$ \\ Frédéric Moisy, ${ }^{1}$ and Pierre-Philippe Cortet ${ }^{1, *}$ \\ ${ }^{1}$ Laboratoire FAST, CNRS, Université Paris-Sud, Université Paris-Saclay, 91405 Orsay, France \\ ${ }^{2}$ Laboratoire LEGI, CNRS, Grenoble INP, Université Grenoble Alpes, 38058 Grenoble, France
}

(Received 20 November 2017; published 9 March 2018)

\begin{abstract}
We analyze theoretically and experimentally the wake behind a horizontal cylinder of diameter $d$ horizontally translated at constant velocity $U$ in a fluid rotating about the vertical axis at a rate $\Omega$. Using particle image velocimetry measurements in the rotating frame, we show that the wake is stabilized by rotation for Reynolds number $\operatorname{Re}=U d / v$ much larger than in a nonrotating fluid. Over the explored range of parameters, the limit of stability is $\operatorname{Re} \simeq(275 \pm 25) / \operatorname{Ro}$, with $\operatorname{Ro}=U / 2 \Omega d$ the Rossby number, indicating that the stabilizing process is governed by the Ekman pumping in the boundary layer. At low Rossby number, the wake takes the form of a stationary pattern of inertial waves, similar to the wake of surface gravity waves behind a ship. We compare this steady wake pattern to a model, originally developed by Johnson [E. R. Johnson, J. Fluid Mech. 120, 359 (1982)], assuming a free-slip boundary condition and a weak streamwise perturbation. Our measurements show quantitative agreement with this model for Ro $\lesssim 0.3$. At larger Rossby number, the phase pattern of the wake is close to the prediction for an infinitely small line object. However, the wake amplitude and phase origin are not correctly described by the weak-streamwise-perturbation model, calling for an alternative model for the boundary condition at moderate rotation rate.
\end{abstract}

DOI: 10.1103/PhysRevFluids.3.034801

\section{INTRODUCTION}

Since the celebrated work of Taylor [1], it has been known that a solid object in slow horizontal motion in a fluid rapidly rotating about the vertical axis tends to drive with it a vertical column of fluid circumscribing the object, as if they were forming together a solid body. The fluid outside of this "Taylor column" flows around it, remaining in the same horizontal plane. This is consistent with the Taylor-Proudman theorem, which states that linear and inviscid fluid motions associated with time scales much longer than the global rotation period must be vertically invariant [2]. Another major feature of rotating fluids is their ability to propagate a specific class of waves, called inertial waves, which are both anisotropic and dispersive [2]. Here we study the flow around a two-dimensional (2D) horizontally invariant cylinder in horizontal translation. This geometry is of interest because the Taylor-column solution is prevented by mass conservation even in the limit of large rotation rate (the fluid cannot flow around the cylinder), so only the inertial wave solution is expected for the wake. The aim of this paper is to compare velocity measurements in this configuration with a theoretical approach based on an approximation of weak streamwise perturbation, originally proposed by Johnson [3]. Although this approximation may seem unnatural for nonslender bodies, it can be shown to apply in the limit of strong background rotation and is indeed found to compare well with our measured wake patterns in this regime.

*ppcortet@fast.u-psud.fr 
The general problem of an object moving horizontally in a fluid under rotation has received a great deal of attention [3-12]. Several flow regimes have been reported depending on the relative importance of rotation, nonlinearities, and viscous effects, characterized by the Rossby number Ro $=U / 2 \Omega L$ and the Reynolds number $\operatorname{Re}=U L / v$, but also on the object height relative to the fluid depth $h / H$ and the object aspect ratio $h / L(\Omega$ is the rotation rate, $U$ the object velocity, and $L$ its size in the streamwise direction). In the strong rotation limit Ro $\ll 1$, the flow can be of two kinds, depending on the geometry: either (i) a geostrophic (nearly) vertically invariant horizontal flow, i.e., the Taylor-column flow [4-7], which cannot be decomposed in terms of inertial waves, or (ii) a wake of inertial waves [3,5-12]. This second kind, akin to the wake of gravity surface waves behind a ship [13,14], is expected when the fluid flows, at least partially, over and/or below the object, locally inducing a vertical velocity perturbation which triggers the emission of inertial waves. The phase configuration of such wake of inertial waves has been derived theoretically for infinitely small objects by Lighthill [8] and Redekopp [9] and further explored experimentally for a sphere by Hide et al. [5] (dye observations) and for a cross-stream cylinder by Peat and Stevenson [10] (schlieren observations). In these works, the question of which region of the wake pattern is supplied with energy was however not addressed; to do so, it is necessary to model the wave-field boundary condition close the object.

The duality between the low-Ro Taylor-column solution and the finite-Ro wake of inertial waves has remained unclear for a long time. An important step was made by Hide and Ibbetson [4], who predicted and verified experimentally that, for objects of comparable vertical $h$ and horizontal $L$ sizes, the Taylor column appears when the Rossby number Ro becomes lower than typically $h / H$ (see also Mason and Sykes [6]). Hide and Ibbetson also exhibited experimentally a reduction of this Ro threshold when the Ekman number $\mathrm{Ek}=\mathrm{Ro} / \mathrm{Re}$ becomes larger than $\sim 10^{-3}$ due to the growing role of viscous boundary layers.

A more precise solution to this duality between Taylor column and wake of inertial waves was proposed for slender bodies $(h / L \ll 1)$ by Stewartson and Cheng [7], who demonstrated the bimodal nature of the flow. They predicted theoretically the inviscid flow produced by the horizontal translation of a thin object by taking the limit Ro $\ll 1$ at fixed parameter $H$ Ro $/ L$ and linearizing the inviscid boundary condition using the slender body assumption. Their analysis, though not employing the term, is quasigeostrophic, replacing the horizontal velocity by its geostrophic value in the acceleration term of the horizontal momentum equation, therefore implying a long time scale and a large vertical scale. For an object of comparable horizontal streamwise and cross-stream lengths $L$, the Taylorcolumn flow is dominant when the parameter $H \mathrm{Ro} / L$ is small, whereas the wake of inertial waves dominates when it is large.

Johnson [3] and Cheng and Johnson [11] extended the description of Stewartson and Cheng [7] to a viscous fluid of arbitrary depth. Johnson [3] studied in particular the case of 2D objects, invariant along the horizontal cross-stream direction, and showed that in this geometry the inviscid nonpenetration boundary condition can be simplified even in the case of nonslender objects $(h / L \sim 1)$ with the assumption of weak streamwise perturbation. In this geometry, the cross-stream invariance also prevents the emergence of a Taylor-column flow and therefore leads to a pure wake of inertial waves down to vanishing Rossby number. The analysis of Johnson also shows how the shape and finite size of the 2D object modify the wake pattern compared to that of an infinitely small source.

Heikes and Maxworthy [12] tested experimentally the predictions of Johnson [3] by studying the perturbation of a horizontal flow by a ridge made of a portion of a cylinder. They used aluminium flakes to highlight shear regions but also to draw flow streamlines on long-exposure images. Their observations revealed an upstream shift of the oscillations compared to the theory as well as a wake amplitude smaller than its theoretical prediction. Heikes and Maxworthy did not consider however viscous dissipation in their model and the reasons (nonlinear effects and viscous effects) for the discrepancies of their experiments with theory remained unclear.

Since then, the range of validity of the weak-streamwise-perturbation approximation for a $2 \mathrm{D}$ nonslender object has remained an open question. In this article we provide a quantitative test of this theory by measuring the wake of a horizontal cylinder in a rotating water-filled tank using 
particle image velocimetry. Our theoretical approach retains the weak-streamwise-perturbation and infinite-depth approximations of Johnson [3], but relaxes the quasigeostrophic approximation, shown by Heikes and Maxworthy [12] to hold for small Rossby number only (typically below $10^{-1}$ ). Viscosity effects are considered in the bulk only, while an inviscid free-slip boundary condition is kept along the object. We also address the stability of the wake in terms of the control parameters (Reynolds and Rossby numbers). This problem is related to the question of the separation and stability of boundary layers on nonvertical surfaces in a fluid rotating about the vertical axis.

After recalling the derivation of the phase field of the inviscid wake of inertial waves of a line object in Sec. II A, we derive in Sec. IIB the velocity field of the steady wake of a cylinder of diameter $d$ using the weak-streamwise-perturbation approximation of Johnson [3]. In Sec. III we study experimentally the threshold in Reynolds and Rossby numbers above which the wake becomes unsteady. These data reveal the strong stabilization of the wake by rotation (Sec. IIIB): We show that the wake remains steady up to $\operatorname{Re} \sim 1000$ at $\operatorname{Ro} \sim 0.3$, a value much larger than the onset of the von Kármán vortex street in a nonrotating fluid. We show in Sec. IIIC that the theory of Sec. II B describes quantitatively the steady wake of inertial waves for Ro $\lesssim 0.3$ and for Re ranging from order 1 to $10^{3}$. These measurements show that the inviscid boundary condition associated with the weak-streamwise-perturbation approximation considered here is valid even for a nonslender $2 \mathrm{D}$ object. At larger Rossby numbers, for which the finite size of the object no longer determines the structure of the wave field, we recover experimentally the wake predicted for a line object. However, we show that the weak-streamwise-perturbation approximation does not describe correctly the amplitude and phase origin of the wake. These last observations call for a better understanding of the nature of the boundary layers on the object at moderate rotation rates.

\section{LINEAR WAKE OF A 2D OBJECT IN HORIZONTAL TRANSLATION IN A ROTATING FLUID}

We describe here the wake produced by the translation at constant velocity $\mathbf{U}=U \mathbf{e}_{x}$ of a $2 \mathrm{D}$ object, invariant along $\mathbf{e}_{y}$, in a fluid rotating at rate $\Omega$ about $\mathbf{e}_{z}$. Small perturbations satisfy the linearized Navier-Stokes equation

$$
\partial_{t} \mathbf{u}=-\frac{1}{\rho} \nabla p-2 \boldsymbol{\Omega} \times \mathbf{u}+v \nabla^{2} \mathbf{u},
$$

where $\mathbf{u}=\left(u_{x}, u_{y}, u_{z}\right)$ is the velocity, $p$ the pressure, $\rho$ the fluid density, $v$ the kinematic viscosity, and $\boldsymbol{\Omega}=\Omega \mathbf{e}_{z}$.

The perturbation induced by a local source can be formally described by a localized field of rate of expansion $q(\mathbf{x}, t)$ acting via the continuity equation

$$
\nabla \cdot \mathbf{u}=q .
$$

In the case of an object under translation at velocity $\mathbf{U}=U \mathbf{e}_{x}$, one can write $q(\mathbf{x}, t)=q_{0}\left(\mathbf{x}-U t \mathbf{e}_{x}\right)$. Such a representation was introduced by Miles [15] and Janowitz [16], among others, in the context of internal gravity waves; Voisin [17] discussed in this context the appropriate expression for $q_{0}$ in the weak and strong stratification limits in the case of a sphere.

Using combinations of derivatives of Eqs. (1) and (2), one can derive the equations of propagation of inertial waves forced by $q$,

$$
\begin{gathered}
{\left[\left(\frac{\partial}{\partial t}-v \nabla^{2}\right)^{2} \nabla^{2}+(2 \Omega)^{2} \frac{\partial^{2}}{\partial z^{2}}\right] u_{x}=\left[\left(\frac{\partial}{\partial t}-v \nabla^{2}\right) \frac{\partial}{\partial x}+2 \Omega \frac{\partial}{\partial y}\right]\left(\frac{\partial}{\partial t}-v \nabla^{2}\right) q,} \\
{\left[\left(\frac{\partial}{\partial t}-v \nabla^{2}\right)^{2} \nabla^{2}+(2 \Omega)^{2} \frac{\partial^{2}}{\partial z^{2}}\right] u_{y}=\left[\left(\frac{\partial}{\partial t}-v \nabla^{2}\right) \frac{\partial}{\partial y}-2 \Omega \frac{\partial}{\partial x}\right]\left(\frac{\partial}{\partial t}-v \nabla^{2}\right) q,} \\
{\left[\left(\frac{\partial}{\partial t}-v \nabla^{2}\right)^{2} \nabla^{2}+(2 \Omega)^{2} \frac{\partial^{2}}{\partial z^{2}}\right] u_{z}=\left[\left(\frac{\partial}{\partial t}-v \nabla^{2}\right)^{2}+(2 \Omega)^{2}\right] \frac{\partial}{\partial z} q .}
\end{gathered}
$$


Considering a plane wave of wave vector $\mathbf{k}$ and angular frequency $\sigma$ and equating the left-hand side of (3) to zero leads to the viscous dispersion relation of inertial waves

$$
\sigma=\sigma_{r}+i \sigma_{i}
$$

with

$$
\sigma_{r}=s 2 \Omega \frac{k_{z}}{k}>0
$$

and

$$
\sigma_{i}=v k^{2}
$$

where $\sigma_{r}$ and $\sigma_{i}$ are the real and imaginary parts of $\sigma$, respectively, $k_{z}=\mathbf{k} \cdot \mathbf{e}_{z}, k=|\mathbf{k}|$, and $s=$ $\operatorname{sgn}\left(k_{z}\right)$. Fluid particles in such a propagative inertial wave describe circular translations oriented by $-s \mathbf{k}$ in planes normal to $\mathbf{k}$ [2]. Because the wave is transverse, the vorticity $\boldsymbol{\omega}$, related to the shear between planes of different phase, is parallel to the velocity $\mathbf{u}$; the wave has helicity $\boldsymbol{\omega} \cdot \mathbf{u}$, of sign given by $-s$. We note that such an inertial wave solution, derived here for small perturbations, is also an exact solution of the full nonlinear Navier-Stokes equation. As a consequence, for finite Ro and outside the viscous boundary layers, nonlinearities can affect inertial waves only via triadic interactions $[18,19]$.

\section{A. Inviscid wake of a line object}

Before searching for solutions to (3)-(5), we first determine the lines of constant phase of the linear inviscid wake produced by the translation of a line object. The equations for these lines were first derived by Lighthill (see, for example, [8]) and by Peat and Stevenson [10] for the case of an arbitrary motion in a fluid with both stratification and rotation. The method we use here is identical to the one initially suggested by Lord Kelvin [20] and Havelock [21] to describe the steady wake of surface gravity waves behind a ship (see also $[13,14]$ ).

In the linearized problem, a steady forcing produces a steady wake in the frame moving with the disturbance. The wake can therefore be described as a superposition of inertial waves that correspond to steady perturbations in this moving frame. For a plane wave of wave vector $\mathbf{k}=k\left(\sin \theta \mathbf{e}_{x}+\right.$ $s \cos \theta \mathbf{e}_{z}$ ) (see Fig. 1), this stationarity condition implies that the angular frequency $\sigma$ satisfies

$$
\sigma=\mathbf{k} \cdot \mathbf{U}=k U \sin \theta,
$$

where $\theta \in[0 ; \pi / 2]$. From the inviscid dispersion relation (7), the stationarity condition becomes

$$
\tan \theta=\frac{2 \Omega}{k U}=\frac{1}{\mathrm{Ro}_{k}},
$$

where $\mathrm{Ro}_{k}$ is the Rossby number associated with wave number $k$. This stationary condition associates a single angle $\theta$ with each wave number.

Assuming that the line object radiates inertial waves of any wave number $k$, the position $C(k, \tau, s)$ of the wave packet, carrying wave number $k$ and helicity sign $-s$ and produced at time $t-\tau$, relative to the position $B$ of the line object at current time $t$, is $\mathbf{B C}(k, \tau, s)=\left(\mathbf{c}_{g}-\mathbf{U}\right) \tau$, where

$$
\mathbf{c}_{g}=\frac{2 \Omega}{k} \sin \theta\left(-\cos \theta \mathbf{e}_{x}+s \sin \theta \mathbf{e}_{z}\right)
$$

is the group velocity associated with the wave vector $\mathbf{k}$ (see Fig. 1) [2]. Using the stationarity condition, the coordinates of $C(k, \tau, s)$ relative to the line object at time $t$ can be written as

$$
\begin{gathered}
X(k, \tau, s)=-U \tau\left(1+\sin ^{2} \theta\right), \\
Z(k, \tau, s)=s U \tau \frac{\sin ^{3} \theta}{\cos \theta} .
\end{gathered}
$$




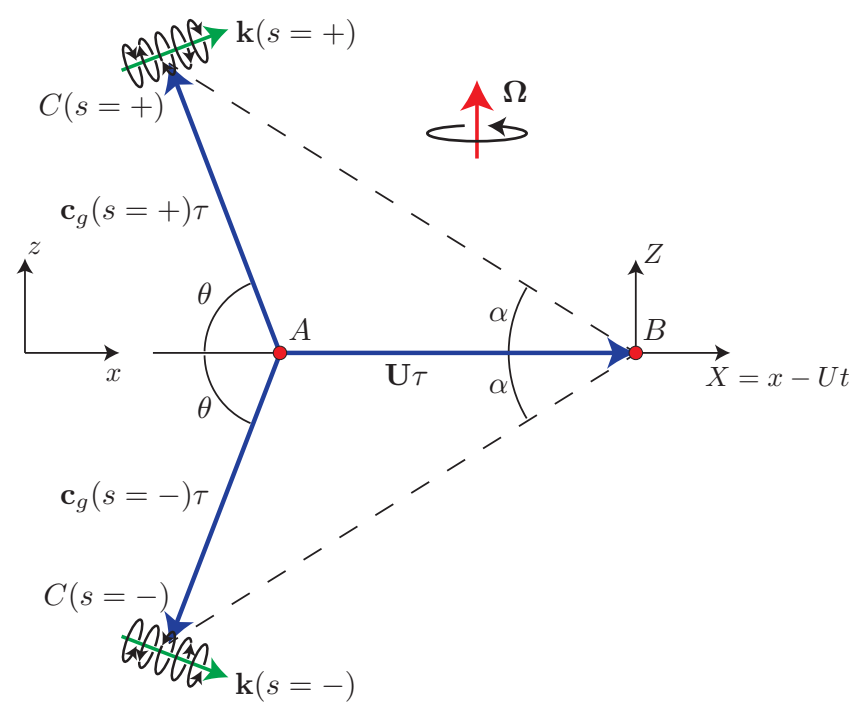

FIG. 1. Propagation of the two symmetric wave packets, carrying wave number $k$, emitted at time $t-\tau$ by a horizontal line object (invariant along $\mathbf{e}_{y}$ ) in translation at constant velocity $\mathbf{U}=U \mathbf{e}_{x}$ in a fluid rotating at a rate $\boldsymbol{\Omega}=\Omega \mathbf{e}_{z}$. At time $t$, the line object is at point $B$, the upper wave packet at point $C(s=+)$, and the lower wave packet at $C(s=-)$. At time $t-\tau$, the line object is at point $A$. At locations $C(s= \pm)$, fluid particles describe a circular translation in planes tilted at an angle $\theta(k)$, with orientation given by the vector $-s \mathbf{k}(s)$. This circular translation motion propagates along wave vector $\mathbf{k}$ at the phase velocity $c_{\varphi}=\sigma / k=U \sin \theta$. Here $(X=x-U t, Z=z)$ is the system of coordinates attached to the moving object.

We can define the radiation angle $\alpha(k)$ as the angle along which energy for a given wave number $k$ is supplied by the disturbance in the moving frame. Writing $\alpha=\tan ^{-1}[|Z(k, \tau) / X(k, \tau)|]$ (see Fig. 1), this angle satisfies

$$
\alpha=\tan ^{-1}\left(\frac{\sin ^{3} \theta}{\cos \theta\left(1+\sin ^{2} \theta\right)}\right)=\tan ^{-1}\left(\frac{1}{2 \mathrm{Ro}_{k}+\mathrm{Ro}_{k}^{3}}\right) .
$$

The line at angle $\alpha(k)$ therefore corresponds to a line of constant wave vector $\mathbf{k}$ (constant $\theta$ and $k$ ). The radiation angle $\alpha(k)$ decreases monotonically with the wave number $k$ from $90^{\circ}$ to $0^{\circ}$ [see Fig. 2(a)]; smaller wavelengths are found closer to the translation axis $z=0$. This contrasts with the case of surface gravity waves, for which $\alpha(k)$ is maximum at a finite wave number, which defines the famous Kelvin angle of ship wakes [13,22,23].

The phase of the wave at point $C(k, \tau, s)$ can be computed as

$$
\varphi(k, \tau, s)=\varphi_{e}+\sigma \tau-\mathbf{k} \cdot \mathbf{A C},
$$

where $\varphi_{e}$ is the phase of the wave when it is emitted and $\mathbf{A C}=\mathbf{c}_{g} \tau$ is the distance traveled by the wave packet between $t-\tau$ and $t$. The anisotropic dispersion relation of inertial waves imposes that $\mathbf{k} \cdot \mathbf{c}_{g}=0$, so the equation for a line of constant phase $\varphi=\varphi_{0}+\varphi_{e}$ satisfies

$$
\varphi_{0}=2 \Omega \tau \cos \theta .
$$

Injecting (16) in (12)-(13) finally provides a parametric representation of the lines of constant phase,

$$
\begin{gathered}
X=-\lambda_{0} \frac{\varphi_{0}}{2 \pi} \frac{1+\sin ^{2} \theta}{\cos \theta}, \\
Z=s \lambda_{0} \frac{\varphi_{0}}{2 \pi} \frac{\sin ^{3} \theta}{\cos ^{2} \theta},
\end{gathered}
$$


(a)

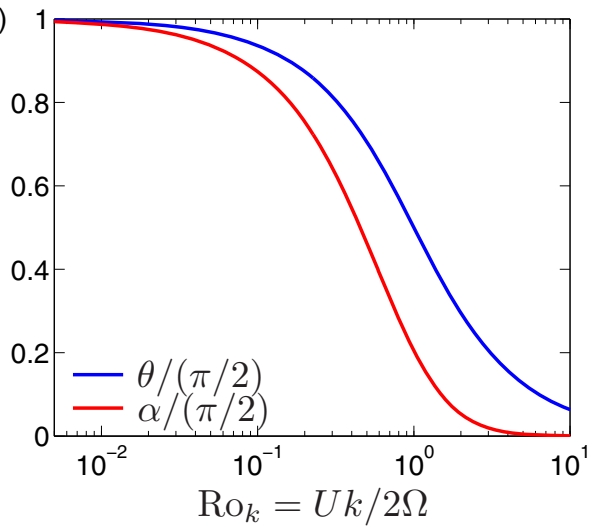

(b)

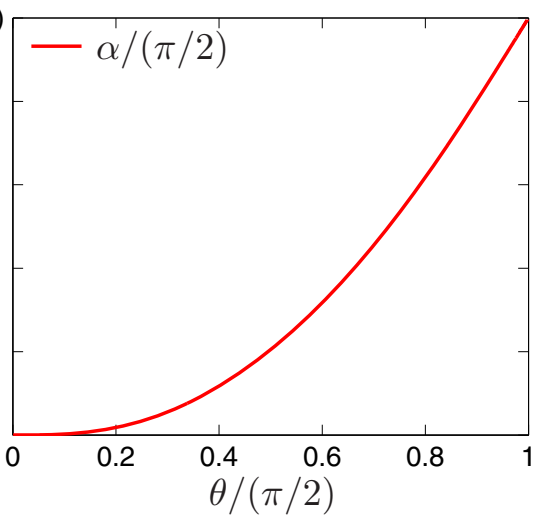

FIG. 2. (a) Energy propagation angle in the frame of the fluid at rest $\theta$ [Eq. (10), blue line] and in the frame of the moving object $\alpha$ [Eq. (14), red line] as a function of the Rossby number $\operatorname{Ro}_{k}=U k / 2 \Omega$ based on the wave number. (b) Plot of $\alpha$ as a function of $\theta$.

with $\lambda_{0}=\pi U / \Omega$ the wavelength of the wake along the translation axis. In Fig. 3(a) we plot the lines of constant phase $\varphi_{0}(X, Z)=2 \pi n$ for $n \in[0 ; 14]$. This phase pattern is identical to that predicted by Lighthill [8] and Mowbray and Rarity [24] for internal waves produced by the vertical translation of a line or point source in a density-stratified fluid (see also Refs. [25-28]). Note that $\varphi_{0}$ is the phase relative to the phase at emission $\varphi_{e}$, which remains unspecified here.

\section{B. Viscous wake of a finite-size object}

We now describe the wake of inertial waves of a translating object of finite size, invariant along $y$, including viscosity effects. For this, we integrate (3)-(5) accounting for the geometry of the object by its equivalent field of rate of expansion $q(\mathbf{x}, t)=q_{0}\left(\mathbf{x}-U t \mathbf{e}_{x}\right)$. The following derivation leads to

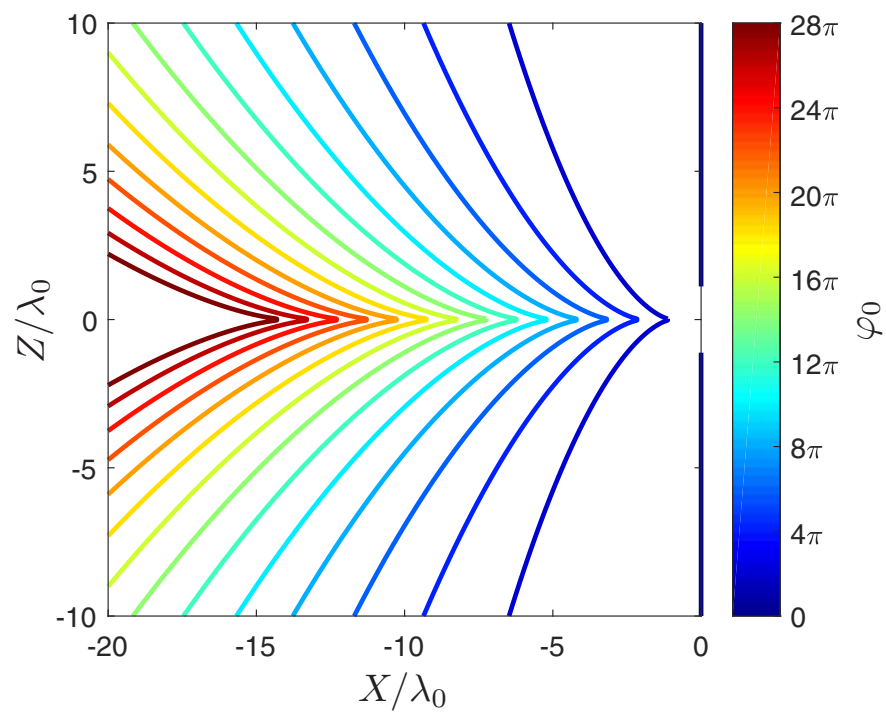

FIG. 3. Lines of constant phase $\varphi_{0}=2 \pi n$ [Eqs. (17) and (18)] with $n \in[0 ; 14]$ in the wake of inertial waves of a line source (of axis $\mathbf{e}_{y}$ ) translated at velocity $U$ along $\mathbf{e}_{x}$ in an inviscid fluid under rotation at a rate $\Omega$ about $\mathbf{e}_{z}$. Coordinates are normalized by $\lambda_{0}=U \pi / \Omega$, the wake wavelength along the translation axis. 
results similar to those in Refs. [3,7,11,12] but differs in the combination of approximations used: infinite depth, free-slip boundary, weak streamwise perturbation, and no quasigeostrophy. Their outcome will be compared to experimental wakes in Sec. III.

We first introduce the 3D spatiotemporal Fourier transform of the field of rate of expansion $q(\mathbf{x}, t)$ invariant along $y$,

$$
\begin{gathered}
\hat{q}(\mathbf{k}, \sigma)=\int q(\mathbf{x}, t) e^{i(\sigma t-\mathbf{k} \cdot \mathbf{x})} d t d x d z, \\
q(\mathbf{x}, t)=\frac{1}{(2 \pi)^{3}} \int \hat{q}(\mathbf{k}, \sigma) e^{-i(\sigma t-\mathbf{k} \cdot \mathbf{x})} d \sigma d k_{x} d k_{z},
\end{gathered}
$$

where $\mathbf{k}=\left(k_{x}, 0, k_{z}\right)$. From Eq. (3)-(5) and using the relation $\hat{q}(\mathbf{k}, \sigma)=2 \pi \hat{q}_{0}(\mathbf{k}) \delta(\sigma-\mathbf{k} \cdot \mathbf{U})=$ $2 \pi \hat{q}_{0}(\mathbf{k}) \delta\left(\sigma-k_{x} U\right)$, which accounts for the stationarity of the forcing in the frame of the translating object, the velocity field is written

$$
\begin{aligned}
& u_{x}(\mathbf{X})=-\frac{i}{(2 \pi)^{2}} \int \frac{\left(U k_{x}+i \nu k^{2}\right)^{2} k_{x}}{\left(U k_{x}+i \nu k^{2}\right)^{2} k^{2}-(2 \Omega)^{2} k_{z}^{2}} \hat{q}_{0}(\mathbf{k}) e^{i \mathbf{k} \cdot \mathbf{X}} d k_{x} d k_{z}, \\
& u_{y}(\mathbf{X})=-\frac{1}{(2 \pi)^{2}} \int \frac{2 \Omega\left(U k_{x}+i \nu k^{2}\right) k_{x}}{\left(U k_{x}+i \nu k^{2}\right)^{2} k^{2}-(2 \Omega)^{2} k_{z}^{2}} \hat{q}_{0}(\mathbf{k}) e^{i \mathbf{k} \cdot \mathbf{X}} d k_{x} d k_{z}, \\
& u_{z}(\mathbf{X})=-\frac{i}{(2 \pi)^{2}} \int \frac{\left[\left(U k_{x}+i v k^{2}\right)^{2}-(2 \Omega)^{2}\right] k_{z}}{\left(U k_{x}+i \nu k^{2}\right)^{2} k^{2}-(2 \Omega)^{2} k_{z}^{2}} \hat{q}_{0}(\mathbf{k}) e^{i \mathbf{k} \cdot \mathbf{X}} d k_{x} d k_{z},
\end{aligned}
$$

with $\mathbf{X}=\mathbf{x}-\mathbf{U} t$ the position relative to the object.

Let us introduce the Rossby number $\operatorname{Ro}_{k_{x}}=U k_{x} / 2 \Omega$ and the Reynolds number $\operatorname{Re}_{k_{x}}=U / \nu k_{x}$ associated with the horizontal wave number $k_{x}$. Application of the residue theorem to the integral over $k_{z}$ allows its evaluation in the low-viscosity limit $\operatorname{Re}_{k_{x}} \gg 1$. The integrand has three poles in the half plane $s \mathfrak{I m}\left(k_{z}\right)>0$, with $s=\operatorname{sgn}(z)$, picked by Jordan's lemma. The first two poles

$$
k_{z}=s\left(\frac{U k_{x} \pm 2 \Omega}{v}\right)^{1 / 2} e^{i \pi / 4}=s \sqrt{\frac{2 \Omega}{v}}\left(\operatorname{Ro}_{k_{x}} \pm 1\right)^{1 / 2} e^{i \pi / 4}
$$

where

$$
\begin{aligned}
\left(U k_{x} \pm 2 \Omega\right)^{1 / 2} & =\sqrt{\left|U k_{x} \pm 2 \Omega\right|} \text { for } U k_{x}>\mp 2 \Omega \\
& =i \sqrt{\left|2 \Omega \pm U k_{x}\right|} \text { for } U k_{x}<\mp 2 \Omega
\end{aligned}
$$

correspond to the boundary layer along the object; we note that the modulus of these two poles (24) tends toward the inverse of the thickness $\sqrt{v / 2 \Omega}$ of an Ekman layer when the Rossby number $\operatorname{Ro}_{k_{x}}$ vanishes. The third pole

$$
k_{z}=k_{z, r}\left(k_{x}\right)+i k_{z, i}\left(k_{x}\right)
$$

of inviscid real part

$$
k_{z, r}=s \frac{U k_{x}\left|k_{x}\right|}{\left[(2 \Omega)^{2}-\left(U k_{x}\right)^{2}\right]^{1 / 2}}=s \frac{\left|k_{x}\right|}{\left(1 / \operatorname{Ro}_{k_{x}}^{2}-1\right)^{1 / 2}}
$$

and viscous imaginary part

$$
k_{z, i}=s \frac{(2 \Omega)^{4} \nu k_{x}^{2}\left|k_{x}\right|}{\left[(2 \Omega)^{2}-\left(U k_{x}\right)^{2}\right]^{5 / 2}}=\frac{s}{\operatorname{Re}_{k_{x}}} \frac{\left|k_{x}\right|}{\operatorname{Ro}_{k_{x}}^{4}\left(1 / \operatorname{Ro}_{k_{x}}^{2}-1\right)^{5 / 2}},
$$


where

$$
\begin{array}{rlrl}
{\left[(2 \Omega)^{2}-\left(U k_{x}\right)^{2}\right]^{1 / 2}} & =\sqrt{\left|(2 \Omega)^{2}-\left(U k_{x}\right)^{2}\right|} \quad \text { when } \quad U\left|k_{x}\right|<2 \Omega \\
& =-\mathrm{i} \sqrt{\left|\left(U k_{x}\right)^{2}-(2 \Omega)^{2}\right|} \operatorname{sgn}\left(k_{x}\right) & \text { when } & U\left|k_{x}\right|>2 \Omega,
\end{array}
$$

corresponds to waves slowly dissipated by viscosity as they propagate away from the object. We note that the spatial decay factor of the wake component associated with $k_{x}$

$$
k_{z, i} Z=s \frac{v}{2 \Omega} \frac{k_{r}^{5}}{k_{x}^{2}} Z,
$$

where $k_{r}^{2}=k_{x}^{2}+k_{z, r}^{2}$ can be written as $\nu k_{r}^{2} \tau$, where $\tau$ is the time for the wave-packet carrying wave vector $\left(k_{x}, k_{z, r}\right)$ to travel from the object to $\mathbf{X}$ at the group velocity. This viscous decay could already have been derived in Sec. II A from the temporal decay due to viscosity of the wave amplitude in the wave-packet carrying wave number $k_{r}$. The determination of the complex square roots (25) and (29) may be seen as the insertion, in the complex $k_{x}$ plane, of branch cuts extending from the singularities $k_{x}= \pm 2 \Omega / U$ vertically downward.

We note that Eq. (27) is identical to the wave stationarity condition (10), with $\operatorname{sgn}(z)$ playing here the same role as $\operatorname{sgn}\left(k_{z}\right)$ there. From Eq. (27), it is apparent that horizontal wave numbers $k_{x}$ larger than $k_{0}=2 \Omega / U$ in absolute value will not contribute significantly to the wake of inertial waves since, to leading order in $1 / \operatorname{Re}_{k_{x}}$, the associated vertical wave number $k_{z}$ is imaginary and leads to vertically evanescent waves. It is worth noting that, contrary to Stewartson and Cheng [7], Johnson [3], and Cheng and Johnson [11], but as in Heikes and Maxworthy [12], no quasigeostrophic approximation of weak vertical derivatives $k_{z} \ll k_{x}$ (i.e., $\mathrm{Ro}_{k_{x}}=U k_{x} / 2 \Omega \ll 1$ ) is made here. The only approximations up to now are the linearization of the Navier-Stokes equation by assuming small velocity perturbations and low viscosity.

In the experiments reported in Sec. III, we consider the translation of a cylinder of diameter $d$ associated with Reynolds numbers $\operatorname{Re}=U d / v$ typically ranging from 10 to 1000 and Rossby numbers Ro $=U / 2 \Omega d$ ranging from $10^{-2}$ to 1 . The Ekman boundary layer on the cylinder, of typical thickness $\sqrt{v / 2 \Omega}=d \sqrt{\mathrm{Ro} / \mathrm{Re}}$, is therefore expected to remain small compared to the cylinder for most of our experiments and the contribution of the corresponding poles in the integration of (21)-(23) will be neglected in the following. Retaining only the pole (26)-(28), the velocity field follows as

$$
\begin{gathered}
u_{x}=-\frac{1}{4 \pi} \int \frac{U\left|k_{x}\right|}{\left[(2 \Omega)^{2}-\left(U k_{x}\right)^{2}\right]^{1 / 2}} \hat{q}_{1}\left(k_{x}\right) e^{-k_{z, i}\left(k_{x}\right) Z} e^{i\left[k_{x} X+k_{z, r}\left(k_{x}\right) Z\right]} d k_{x}, \\
u_{y}=\frac{i}{4 \pi} \int \frac{2 \Omega \operatorname{sgn}\left(k_{x}\right)}{\left[(2 \Omega)^{2}-\left(U k_{x}\right)^{2}\right]^{1 / 2}} \hat{q}_{1}\left(k_{x}\right) e^{-k_{z, i}\left(k_{x}\right) Z} e^{i\left[k_{x} X+k_{z, r}\left(k_{x}\right) Z\right]} d k_{x}, \\
u_{z}=\frac{\operatorname{sgn}(Z)}{4 \pi} \int \hat{q}_{1}\left(k_{x}\right) e^{-k_{z, i}\left(k_{x}\right) Z} e^{i\left[k_{x} X+k_{z, r}\left(k_{x}\right) Z\right]} d k_{x},
\end{gathered}
$$

where $X=x-U t, Z=z$, and $\hat{q}_{1}\left(k_{x}\right)=\hat{q}_{0}\left(k_{x}, k_{z, r}\left(k_{x}\right)\right)$.

\section{Far-field limit}

In the far-field limit $k_{0}|\mathbf{X}| \gg 1$, the integrals over $k_{x}$ (31)-(33) may be evaluated asymptotically. In the inviscid case, the phase of the integrand is real in the range $\left|k_{x}\right|<k_{0}$ of propagating waves and the stationary phase method may be applied (see Appendix A). The waves are only found downstream, in the half plane $X<0$. There, two opposite stationary points $k_{x}= \pm k_{0} \cos \theta$ are obtained for each radiation angle $\alpha=\tan ^{-1}(|Z / X|)$, the angle $\theta$ satisfying the cubic equation

$$
\cot ^{3} \theta+2 \cot \theta-\cot \alpha=0,
$$


consistent with (14), of real root

$$
\cot \theta=\left(\sqrt{\frac{\cot ^{2} \alpha}{4}+\frac{8}{27}}+\frac{\cot \alpha}{2}\right)^{1 / 3}-\left(\sqrt{\frac{\cot ^{2} \alpha}{4}+\frac{8}{27}}-\frac{\cot \alpha}{2}\right)^{1 / 3}
$$

The associated wave vectors are $\pm \mathbf{k}_{s}$, with

$$
\mathbf{k}_{s}=k_{0} \cot \theta\left(\sin \theta \mathbf{e}_{x}+s \cos \theta \mathbf{e}_{z}\right),
$$

consistent with (10), and we also recover the same phase field as the one described in Sec. II A.

Viscosity adds a small imaginary part to the phase of the integrand in (31)-(33). To leading order in $1 / \operatorname{Re}\left(\right.$ see Appendix A), the result is a slow exponential decay as $\exp \left(-k_{z, i} Z\right)=$ $\exp \left[-k_{0}|Z| \cos ^{3} \theta /\left(\operatorname{Re} \operatorname{Ro} \sin ^{5} \theta\right)\right]$. The far-field velocity follows as

$$
\begin{aligned}
& u_{x}=-H(-X) \frac{\sin ^{3 / 2} \theta \cos \theta}{\sqrt{2+\cos ^{2} \theta}} \exp \left(-\frac{k_{0}|Z|}{\operatorname{Re} \operatorname{Ro}} \frac{\cos ^{3} \theta}{\sin ^{5} \theta}\right) \frac{\mathfrak{R e}\left[\hat{q}_{0}\left(\mathbf{k}_{s}\right) e^{-i \varphi_{s}}\right]}{\sqrt{\lambda_{0}|Z|}} \\
& u_{y}=-H(-X) \frac{\sin ^{3 / 2} \theta}{\sqrt{2+\cos ^{2} \theta}} \exp \left(-\frac{k_{0}|Z|}{\operatorname{Re} \operatorname{Ro}} \frac{\cos ^{3} \theta}{\sin ^{5} \theta}\right) \frac{\mathfrak{I m}\left[\hat{q}_{0}\left(\mathbf{k}_{s}\right) e^{-i \varphi_{s}}\right]}{\sqrt{\lambda_{0}|Z|}} \\
& u_{z}=H(-X) \frac{\sin ^{5 / 2} \theta \operatorname{sgn}(Z)}{\sqrt{2+\cos ^{2} \theta}} \exp \left(-\frac{k_{0}|Z|}{\operatorname{Re} \operatorname{Ro}} \frac{\cos ^{3} \theta}{\sin ^{5} \theta}\right) \frac{\mathfrak{R e}\left[\hat{q}_{0}\left(\mathbf{k}_{s}\right) e^{-i \varphi_{s}}\right]}{\sqrt{\lambda_{0}|Z|}},
\end{aligned}
$$

where $H$ denotes the Heaviside step function, $\mathfrak{R e}$ and $\mathfrak{I m}$ are the real and imaginary parts, respectively, and

$$
\varphi_{s}=\underbrace{k_{0}|Z| \frac{\cos ^{2} \theta}{\sin ^{3} \theta}}_{=\varphi_{0}} \underbrace{-\frac{\pi}{4}}_{=\varphi_{e}} .
$$

In this expression, the first term $k_{0}|Z| \cos ^{2} \theta / \sin ^{3} \theta$ corresponds to the phase field $\varphi_{0}$ derived in Sec. II A, while the second term allows us to identify the value of the phase at emission, $\varphi_{e}=-\pi / 4$, which was left unknown in Sec. II A. The lines of constant phase $\varphi_{s}=$ const have the parametric equation

$$
\begin{gathered}
k_{0}|X|=\frac{1+\sin ^{2} \theta}{\cos \theta}\left(\varphi_{s}+\frac{\pi}{4}\right), \\
k_{0}|Z|=\frac{\sin ^{3} \theta}{\cos ^{2} \theta}\left(\varphi_{s}+\frac{\pi}{4}\right),
\end{gathered}
$$

consistent with (17) and (18). Johnson [3] pointed out that, as Re decreases to order unity, viscosity also induces a deformation of these lines. This effect is discussed in Appendix B.

\section{Boundary condition model for the object spectrum}

We finally introduce a model for the spectrum $\hat{q}_{1}\left(k_{x}\right)$ of the equivalent source of inertial waves induced by the motion of the object. Since we neglect here the Ekman boundary layer on the object surface, we use an inviscid nonpenetration boundary condition as in Refs. [7,11,12],

$$
(\mathbf{u}-\mathbf{U}) \cdot \mathbf{n}=0,
$$

where $\mathbf{n}$ is the vector normal to the object surface and $\mathbf{u}-\mathbf{U}$ the fluid velocity in the reference frame of the object. We consider in the following a symmetric object, of boundary described by 
$Z= \pm f(X)$, yielding a boundary condition

$$
u_{z}= \pm\left(u_{x}-U\right) \frac{d f}{d X}
$$

We are here mainly interested in wakes at small Rossby number, for which finite-size effects are expected ( $\mathrm{Ro}=U / 2 \Omega d$ compares the size $d$ of the object to the characteristic wavelength $\lambda_{0}=\pi U / \Omega$ of the inviscid wake for a line object). In this regime, the horizontal wave numbers of the order of $k_{x} \sim 2 \pi / d$, which are associated with small $\operatorname{Ro}_{k_{x}}=U k_{x} / 2 \Omega$, are expected to dominate the wake. From Eq. (27), the leading spectral components verify $\left|k_{z, r} Z\right| \simeq \operatorname{Ro}_{k_{x}}\left|k_{x} Z\right| \ll 1$ for $|Z| \leqslant d$. Accordingly, the phase of the dominant components in the integrand of (31)-(33) shows weak variations over the vertical extent $|Z| \leqslant d$ of the object, so it is justified to apply the boundary condition (44) at $Z=0_{ \pm}$instead of $Z= \pm f(X)$.

In the following, we consider an object invariant along $y$, of aspect ratio of order 1 in the vertical plane. Still assuming small Rossby number Ro $=U / 2 \Omega d$, the stationarity condition (10) [or equivalently Eqs. (27) and (31)-(33)] suggests that the ratio $u_{x} / u_{z}$ of the axial to vertical velocity perturbations due to the leading spectral terms of the wake, which is given by $1 / \tan \theta=\mathrm{Ro}_{k} \simeq \mathrm{Ro}_{k_{x}}$, is small as well. We will therefore assume in Eq. (44) that the horizontal velocity $u_{x}$ is negligible with respect to $u_{z}$ close to the cylinder, $u_{z}$ being itself assumed to be of order $U$ because of the aspect ratio 1 of the object; this constitutes the assumption of weak streamwise perturbation at the core of the present model.

In a nonrotating fluid, the approximation $u_{x} \ll U$ is relevant only for slender bodies verifying $d f / d X \ll 1$. Here it also applies for a bluff body because of the small value of the Rossby number, provided the body is invariant along $y$, as first noted by Johnson [3] and Heikes and Maxworthy [12]. This can be understood by eliminating the pressure term in the expressions of the 4D spatiotemporal Fourier transform of the inviscid and linearized Navier-Stokes equation, which leads to

$$
\begin{gathered}
\hat{u}_{x}=i \frac{k_{z}}{k} \hat{u}_{y}-i \frac{k_{y}}{k} \hat{u}_{z}, \\
\hat{u}_{y}=-i \frac{k_{z}}{k} \hat{u}_{x}+i \frac{k_{x}}{k} \hat{u}_{z} .
\end{gathered}
$$

For small Rossby numbers $\operatorname{Ro}_{k_{x}}$, the wave stationarity condition gives $k_{z} / k \simeq \operatorname{Ro}_{k_{x}}$ and $k_{x} / k \simeq$ $O$ (1). Equations (45) and (46) therefore show that $u_{x} / u_{z}=O\left(\operatorname{Ro}_{k_{x}}\right)$ if $k_{y}=0$, whereas $k_{y} \sim k_{x}$ and $u_{x} / u_{z}=O(1)$ for a $3 \mathrm{D}$ object of aspect ratio of order 1 .

Under these approximations, the boundary condition (44) is simply written

$$
u_{z}=\mp U \frac{d f}{d X} \quad \text { at } Z=0_{ \pm}
$$

This velocity discontinuity implies a rate of expansion

$$
q_{0}(X, Z)=\left[u_{z}\left(X, Z=0_{+}\right)-u_{z}\left(X, Z=0_{-}\right)\right] \delta(Z)=-2 U f^{\prime}(X) \delta(Z),
$$

leading to

$$
q_{1}(X)=-2 U f^{\prime}(X),
$$

which is consistent with Eq. (33) applied at $Z=0_{ \pm}$. In the following, we consider a cylinder of diameter $d=2 R$ such that $f(X)=H(R-|X|) \sqrt{R^{2}-X^{2}}$, where $H$ is the Heaviside step function. The boundary condition is written

$$
u_{z}\left(X, Z=0_{ \pm}\right)= \pm U \frac{X}{\sqrt{R^{2}-X^{2}}} H(R-|X|),
$$



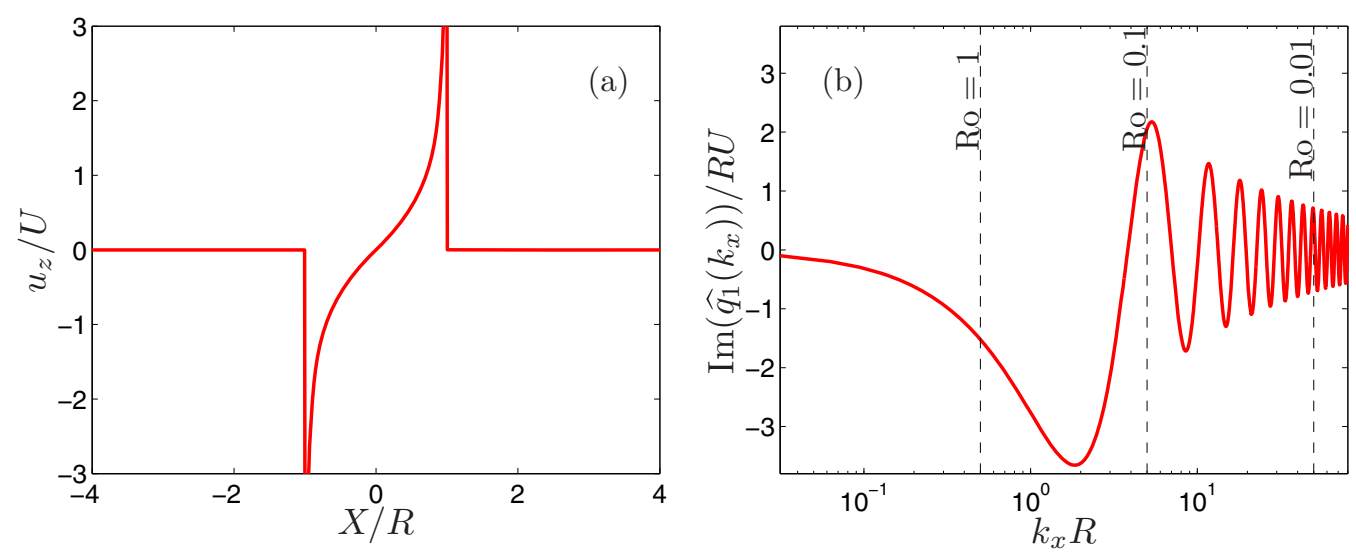

FIG. 4. (a) Vertical velocity profile $u_{z}(X) / U$ at $Z=0_{+}$used as the boundary condition (50) in the weakstreamwise-perturbation approximation. (b) Corresponding spectrum $\mathfrak{I m}\left[\hat{q}_{1}\left(k_{x}\right)\right] / R U$ [Eq. (51)]. We report with vertical dashed lines the critical horizontal wave-vector component $k_{0}=1 / \operatorname{Ro} d$, above which waves are vertically evanescent for three values (Ro $=0.01,0.1$, and 1$)$ of the Rossby number Ro $=U / 2 \Omega d$.

yielding the representation

$$
q_{1}(X)=2 U \frac{X}{\sqrt{R^{2}-X^{2}}} H(R-|X|), \quad \hat{q}_{1}\left(k_{x}\right)=-2 i \pi R U J_{1}\left(k_{x} R\right),
$$

where $J_{1}$ is the Bessel function of the first kind of order 1 .

Figure 4 shows the vertical velocity at the boundary (50) and the corresponding spectrum $\hat{q}_{1}\left(k_{x}\right)$ [Eq. (51)]. In Fig. 4(b) we show with vertical dashed lines the critical horizontal wave-vector component $k_{0}=1 /(\operatorname{Ro} d)$, above which waves are vertically evanescent for three values of the Rossby number $(\operatorname{Ro}=0.01,0.1$, and 1$)$; only the portion of the spectrum $\hat{q}_{1}\left(k_{x}\right)$ at wave numbers $k_{x}$ smaller than $k_{0}$ contributes to the wake.

The wake structure, computed from Eqs. (31)-(33) using a fast Fourier transform (FFT) algorithm, is shown in Fig. 5 for two Rossby numbers Ro $=0.02$ and 0.20 and two Reynolds numbers $\operatorname{Re}=$ 26 and $\infty$. In the low-Rossby-number case [Fig. 5(b)], we observe the concentration of energy along a set of radiation angles $\alpha_{\text {extr }}^{n}$ corresponding to the extrema of the Bessel function $\left(k_{x, \text { extr }}^{n} R \simeq\right.$ $1.84,5.33,8.54, \ldots)$, separated by angles $\alpha_{\text {zero }}^{n}$ along which no energy is present (highlighted with dash-dotted lines) corresponding to its roots $\left(k_{x, \text { zero }}^{n} R \simeq 3.83,7.02,10.17, \ldots\right)$. These oscillations are the interference pattern due to the finite size of the object. According to Eqs. (14) and (27), the specific angles $\alpha_{\text {extr }}^{n}$ and $\alpha_{\text {zero }}^{n}$ decrease with the order $n$ and with the cylinder Rossby number Ro. In Fig. 5(b), in each angular sector between successive $\alpha_{\text {zero }}^{n}$, we observe good agreement between the computed velocity field and the lines of constant phase (41) and (42) predicted for a line object; however, we observe a sign change of the velocity perturbation at each $\alpha_{\text {zero }}^{n}$ along the line of constant phase of the line object associated with the corresponding sign change of $\hat{q}_{1}\left(k_{x}\right)$. Looking now at Fig. 5(a) for the same Rossby number as in Fig. 5(b) but for $\mathrm{Re}=26$, we see that, except for the beam corresponding to the first and largest minimum of $\hat{q}_{1}\left(k_{x}\right)$ (at $k_{x, \text { extr }}^{1} R \simeq 1.84$ ), all the beams are rapidly damped by viscosity, leading to a wake of typical angle $\alpha_{\text {extr }}^{1}\left(\simeq 82^{\circ}\right.$ here for Ro $\left.=0.02\right)$ and to a significant discrepancy with the lines of constant phase of the line object.

For $\mathrm{Ro}=\operatorname{Ro}_{c} \equiv 1 / 2 k_{x, \text { zero }}^{1} R \simeq 0.13$, the radiation angle $\alpha_{\text {zero }}^{1}$ associated with the first zero of the spectrum reaches $0^{\circ}$, while the zero itself $k_{x \text {, zero }}^{1} R \simeq 3.83$ matches the critical wave number $k_{0} R=1 / 2$ Ro above which inertial waves are evanescent. As Ro further increases above $\mathrm{Ro}_{c}$, a smaller portion of the spectrum $k_{x}<k_{0}\left(<k_{x, \text { zero }}^{1}\right)$ contributes to the wake, containing no zero. Considering, for example, the wake at Rossby number Ro $=0.20>\mathrm{Ro}_{c}$ in Figs. 5(c) and 5(d), we see that the two Reynolds numbers considered here, $\operatorname{Re}=26$ and $\infty$, lead this time to similar 

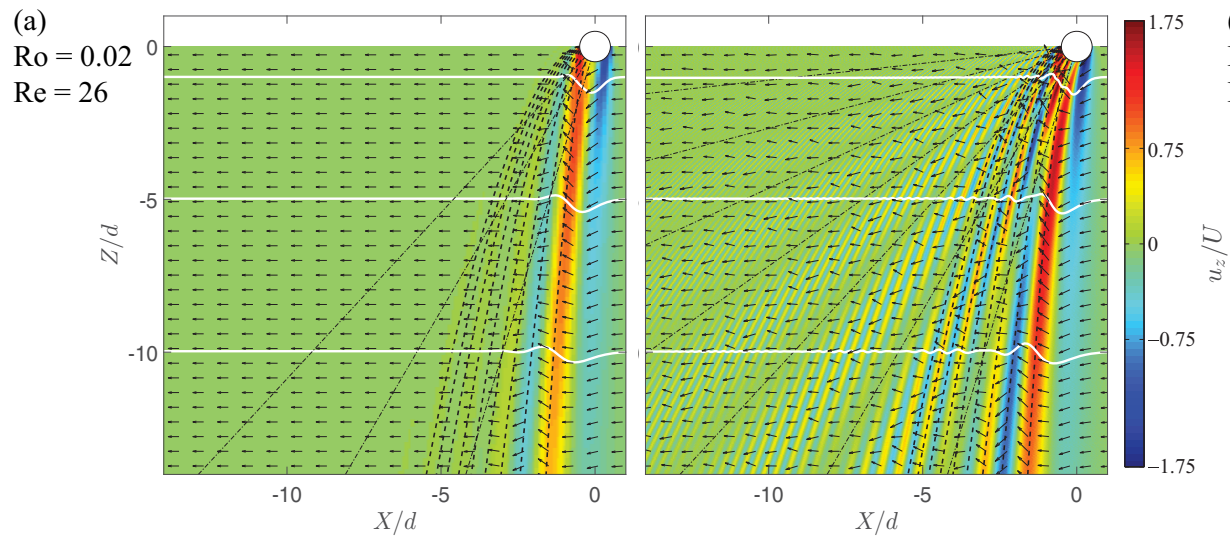

(b)

Ro $=0.02$

$\operatorname{Re}=\infty$
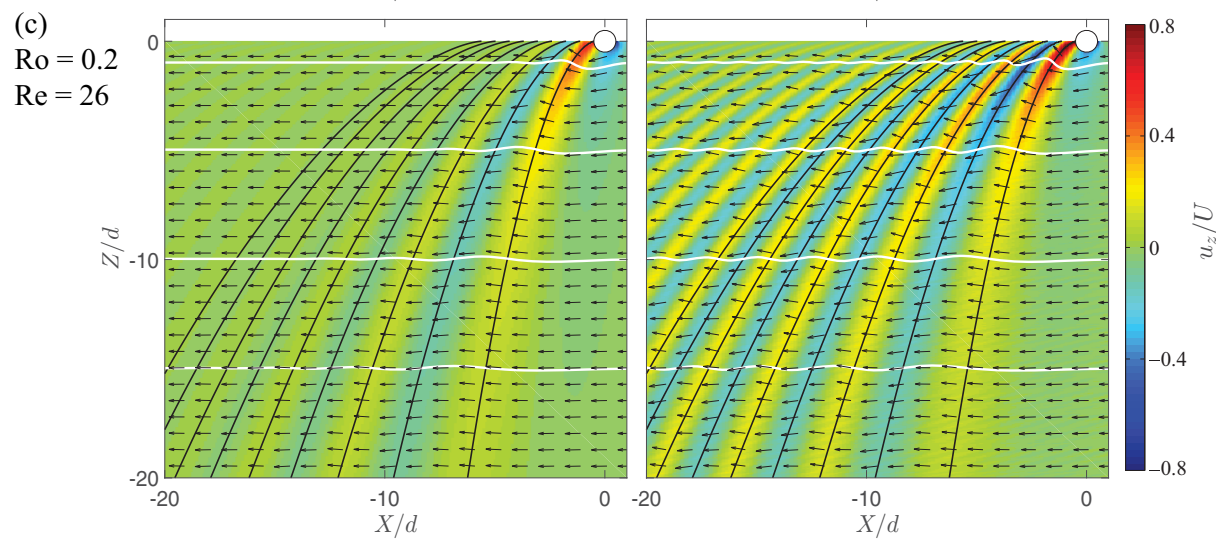

(d)

Ro $=0.2$

$\operatorname{Re}=\infty$

FIG. 5. Wake structure computed from the weak-streamwise-perturbation model (31)-(33) for a cylinder of diameter $d$, invariant along the $y$ direction. Arrows show the in-plane velocity components $\left(u_{x}, u_{z}\right)$ in the reference frame of the cylinder and the colormap the vertical velocity component $u_{z}$ normalized by $U$. White lines show the projection of streamlines in the vertical plane. In (a) and (b) the dash-dotted lines show the radiation angles $\alpha_{\text {zero }}^{n}$, associated with the first roots $k_{x, \text { zero }}^{n} R$ of the spectrum, along which no energy is present. In (c) and (d) these angles of zero amplitude do not exist since the roots of the spectrum $\hat{q}_{1}\left(k_{x}\right)$ correspond to evanescent waves. In (a)-(d) we also show with dashed lines a few lines of constant phase of the far-field wake (37)-(39). We actually plot the parametric curve (41) and (42) for $\varphi_{s}+\pi / 2=\pi, 2 \pi, 3 \pi, 4 \pi, \ldots, 9 \pi$ (from right to left) corresponding to the local maxima and minima of $u_{z}$ in the far-field theory (39) for the spectrum (51) [the spectrum (51) being imaginary introduces the $+\pi / 2$ phase shift].

wake structures which match well the lines of constant phase for a line object (41) and (42). We can conclude that, in this weak-streamwise-perturbation model, $\operatorname{Ro}_{c}=1 / k_{x, \text { zero }}^{1} d \simeq 0.13$ stands as an approximate threshold for the appearance of finite-size effects in the wake of a cylinder. We finally note that, in the finite-Re cases of Figs. 5(a) and 5(c), the wake is damped by viscosity more efficiently as the wake angle $\alpha=\tan ^{-1}(|Z / X|)$ becomes smaller. Recalling that the decay factor for a propagating wave is $\exp \left(-v k^{2} \tau\right)$, this result is consistent with the prediction of a decreasing wave number $k$ with $\alpha$ [Eq. (14)] made in Sec. II A for an infinitely small object. We also note that the wake in Figs. 5(a) and 5(c) is progressively damped along a given direction $\alpha$, in agreement with the fact that it corresponds to an increasing propagation time $\tau$ at constant wave number $k$.

Finally, for the sake of comparison with the full model (31)-(33), we show in Fig. 6 the velocity field predicted in the far-field approximation (37)-(39), still using the weak-streamwise-perturbation spectrum of the cylinder (51), for the same values of Ro and Re as in Fig. 5. One can see that for Ro $=0.2$ the far-field wake in Figs. 6(c) and 6(d) and the one of the full model in Figs. 5(c) and 5(d) 

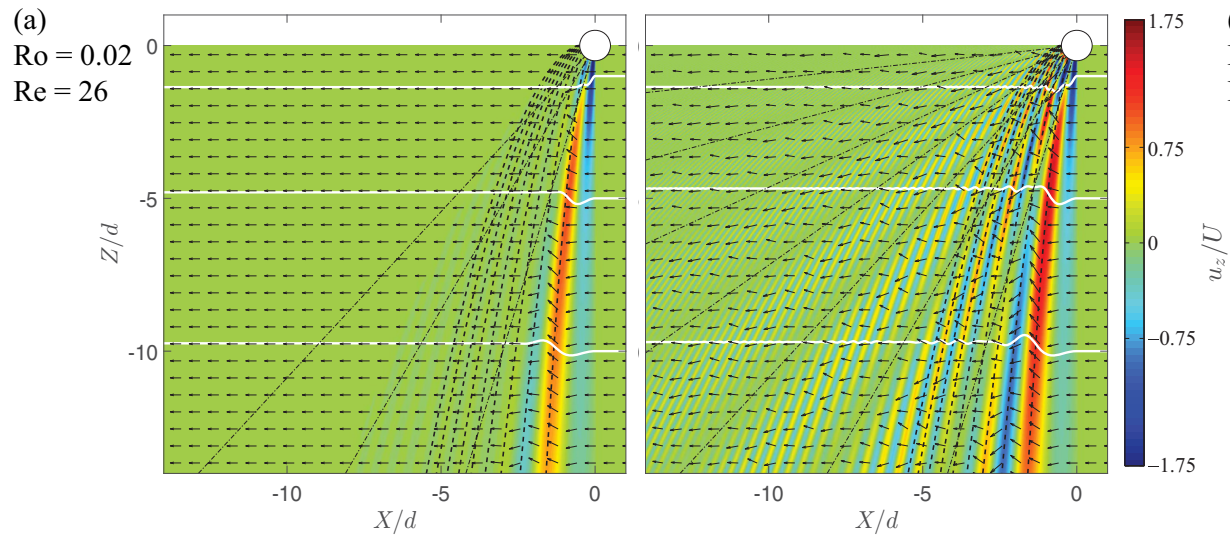

(b)

Ro $=0.02$

$\operatorname{Re}=\infty$
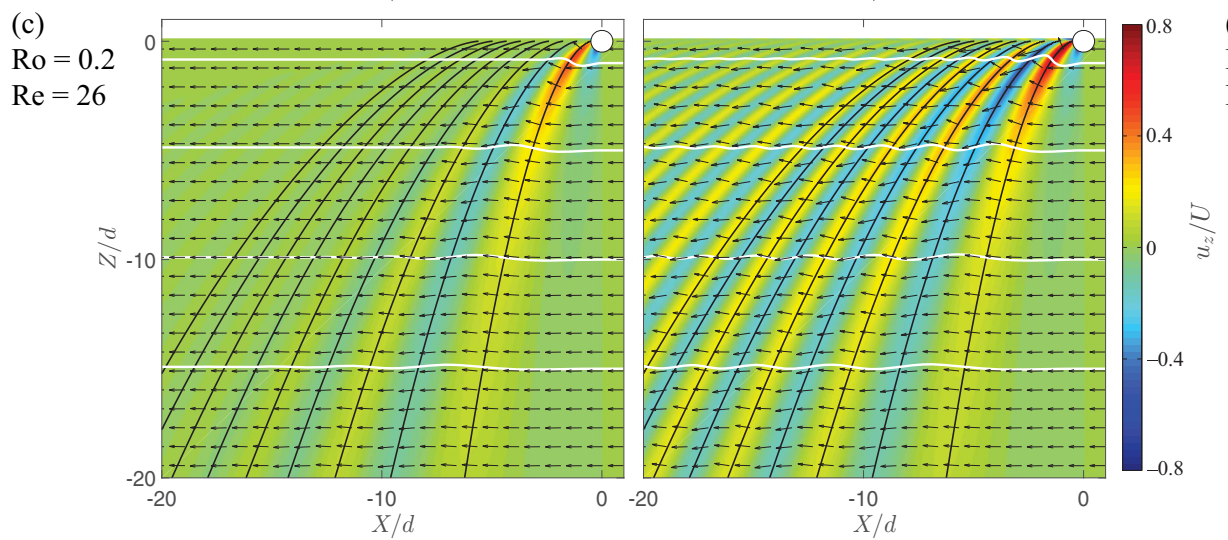

(d)

$\mathrm{Ro}=0.2$

$\operatorname{Re}=\infty$

FIG. 6. Wake structure, for the same values of Ro and Re as in Fig. 5, predicted in the far-field approximation (37)-(39) of the weak-streamwise-perturbation model [spectrum (51)] for a cylinder of diameter $d$, invariant along the $y$ direction. The layout is the same as in Fig. 5 .

are almost identical for distances from the cylinder larger than typically its diameter. On the other hand, comparing now Figs. 6(a) and 6(b) and Figs. 5(a) and 5(b) at Ro $=0.02$ reveals significant differences up to distances much larger than the cylinder diameter, with a much thinner "main beam," the nearly vertical beam found around the wake angle $\alpha_{\text {extr }}^{1} \simeq 82^{\circ}$. We note in particular that the absence of flow perturbation upstream, that is, for $X>0$, in the far-field model constitutes a significant discrepancy with the full model at low Ro.

The absence of upstream flow perturbation in Fig. 6 is a manifestation of the nonuniformity of the expansion (37)-(39) at the wave front $X=0$; there, diffraction takes place, requiring the switch to a uniform expansion valid for all $X$ and involving Fresnel functions. The mathematical origin of the nonuniformity and the derivation of the uniform far-field expansion are briefly discussed in Appendix C. The uniform expansion gives to the wave field some extension upstream, however still smaller than with the full model. Another noticeable feature of expansion (37)-(39), visible in Fig. 6, is an unphysical vertical shift of the streamlines between their original position upstream and their final position downstream. The uniform far-field expansion (C1)-(C3) presented in Appendix $\mathrm{C}$ reduces the vertical shift for most streamlines (see Fig. 16), however not for those close to the cylinder at low Ro (i.e., for Ro $=0.02$ ). The origin of this vertical shift of the streamlines is related to a wrong estimation by the far-field expansion of the velocity perturbation in the near-field region close to the cylinder (which increases in size as Ro decreases) as well as in the region close to the axis $X=0$ for the nonuniform expansion (37)-(39). 


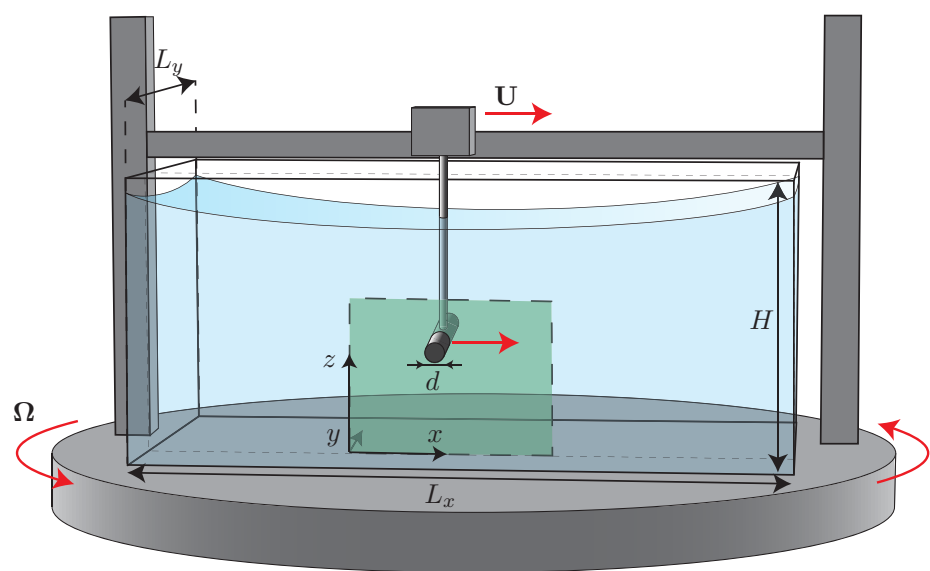

FIG. 7. Experimental setup. A motor coupled by a belt to a translation rail drives a horizontal cylinder, of diameter $d$ and length $65 \mathrm{~cm}$, at a constant velocity $U \mathbf{e}_{x}$ in a parallelepipedic water-filled tank mounted on a platform rotating at a rate $\Omega$ about $z$. The PIV measurements are performed in the rotating frame, in a vertical region of $47 \times 35 \mathrm{~cm}^{2}$ (green area). Here $L_{x}=150 \mathrm{~cm}, L_{y}=80 \mathrm{~cm}$, and $H=65 \mathrm{~cm}$.

Overall, the comparison of Figs. 5 and 6 shows that the full model (31)-(33) should be preferred to fully describe the cylinders' wake for Rossby numbers below typically 0.1 .

\section{EXPERIMENTS}

\section{A. Experimental setup}

The experimental setup is sketched in Fig. 7. A horizontal cylinder, $65 \mathrm{~cm}$ long in the $y$ direction, is towed horizontally along $x$ at constant velocity $U$ between 0.6 and $83 \mathrm{~mm} \mathrm{~s}^{-1}$ using a stepper motor coupled to a translation rail by a belt. The translation motion, $70 \mathrm{~cm}$ long, takes place in a parallelepipedic tank, of base $L_{x} \times L_{y}=150 \times 80 \mathrm{~cm}^{2}$ and height $65 \mathrm{~cm}$, filled with $50 \mathrm{~cm}$ of water. We have used four cylinder diameters $d=4.1,10.1,20.6$, and $40.2 \mathrm{~mm}$. The whole system is mounted on a 2-m-diam platform rotating at a constant rate $\Omega$, in the range $2-20 \mathrm{rpm}$, about the vertical axis $z$. The rotation of the platform is set at least $20 \mathrm{~min}$ before the cylinder translation to avoid transient spin-up recirculations. The ranges of Reynolds and Rossby numbers explored here, shown in Fig. 8, are $0.01 \lesssim$ Ro $\lesssim 20$ and $2 \lesssim \operatorname{Re} \lesssim 3500$.

The two components $\left(u_{x}, u_{z}\right)$ of the velocity field are measured in the vertical plane $y_{0} \simeq L_{y} / 3$ normal to the cylinder axis using a particle image velocimetry (PIV) system mounted in the rotating frame ( $y=0$ is the tank front face, Fig. 7). The fluid is seeded with $10-\mu \mathrm{m}$ tracer particles and illuminated by a laser sheet generated by a corotating $140-\mathrm{mJ} \mathrm{Nd}$ :YAG pulsed laser. Images of particles are acquired with a $2360 \times 1776$ pixel camera in a region of interest of $47 \times 35 \mathrm{~cm}^{2}$. Each PIV acquisition consists of 200-500 images recorded at a rate between 0.5 and $29 \mathrm{~Hz}$ depending on the amplitude of the velocity perturbation induced by the cylinder translation. Cross correlation between successive images, performed over windows of $16 \times 16$ pixels with $50 \%$ overlap, produces velocity fields sampled on a grid of $295 \times 222$ vectors with a spatial resolution of $\sim 1.6 \mathrm{~mm}$. Image acquisition starts after $25 \mathrm{~cm}$ of translation, such that a steady wake regime is reached, and the following $\sim 40 \mathrm{~cm}$ of translation is recorded.

\section{B. Steady vs unsteady wake}

We first determine the nature of the wake as a function of the two control parameters Re and Ro. For each experiment, we remap the velocity field in the frame moving with the cylinder $(X=x-U t, Z=z)$ and subtract the cylinder velocity $U \mathbf{e}_{x}$. From these movies (see the 


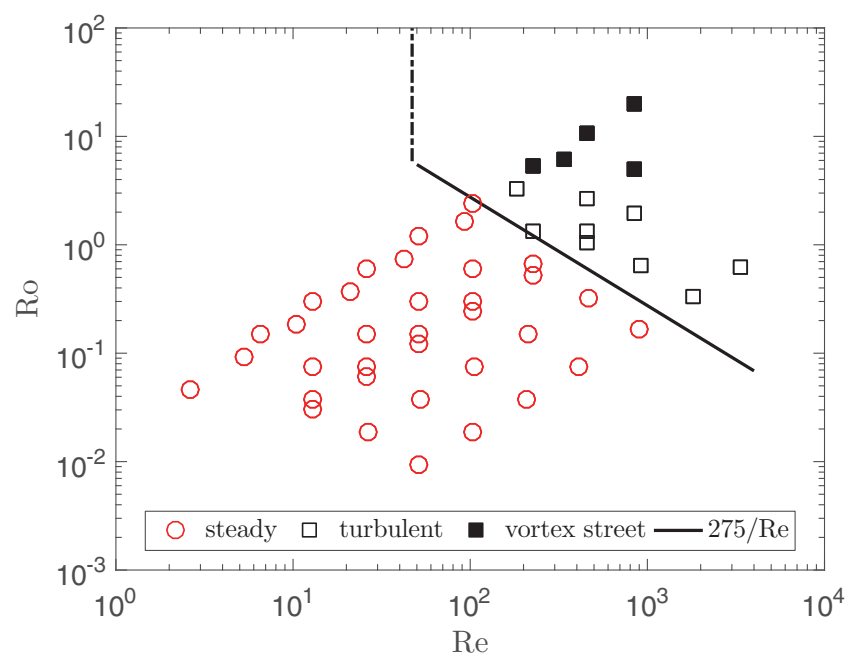

FIG. 8. Stability diagram of the cylinder wake in the (Re,Ro) plane: ॰, steady wake; $\mathbf{\square}$, periodic vortex shedding; and $\square$, turbulent wake. The solid line delimits the transition to unsteadiness, Ro $=275 /$ Re. The vertical dash-dotted line shows the classical instability threshold in a nonrotating fluid, $\operatorname{Re}=47$.

Supplemental Material [29]) we classify the wakes in three categories, summarized in Fig. 8: steady, unsteady with periodic vortex shedding, and turbulent.

The most remarkable effect of the global rotation is the stabilization of the steady wake: In a nonrotating fluid $(\operatorname{Ro}=\infty)$, the wake becomes unsteady for $\operatorname{Re}>\operatorname{Re}_{c}(\infty) \simeq 47$ through the von Kármán vortex shedding phenomenon [30,31], whereas here steady wakes are found up to $\mathrm{Re} \simeq 1000$ for the largest rotation rate. It is worth highlighting that a similar stabilization of the wake is observed for a horizontal cylinder translated horizontally in a linearly stratified fluid [32,33].

An approximate Rossby-number dependence of the critical Reynolds number for unsteadiness can be inferred from our data, $\operatorname{Re}_{c}=(275 \pm 25) / \mathrm{Ro}$, indicating that the stability of the wake is governed here by the combination Re Ro. This stability criterion suggests the following scenario. In a nonrotating fluid, stability is ensured by viscous diffusion: Separation of the boundary layer and subsequent instability of the detached layer occur when the inertial time scale $\tau_{i}=d / U$ becomes shorter than the viscous time scale $\tau_{v}=d^{2} / \nu$, yielding $\tau_{d} / \tau_{i}=\operatorname{Re}$ as the natural control parameter. In a rotating fluid, Ekman pumping, here at the surface of the cylinder, provides an effective diffusion mechanism on a shorter-time scale, given by the Ekman time scale $\tau_{\mathrm{Ek}}=d / \sqrt{v 2 \Omega}$ [2]. Balancing $\tau_{\mathrm{Ek}}$ and $\tau_{i}$ now yields $\tau_{\mathrm{Ek}} / \tau_{i}=\sqrt{\operatorname{Re} \mathrm{Ro}}$ as the new control parameter, in good agreement with our data.

Close-up views of the flow near the cylinder, as shown in Fig. 9, confirm the inhibition due to rotation of the separation of the viscous boundary layers from the cylinder. Although the PIV resolution $(1.6 \mathrm{~mm})$ is of the same order of magnitude as the thickness of the Ekman boundary layer $\delta \simeq \sqrt{v / 2 \Omega}$, good insight into the nature of the flow is provided by plotting the streamlines, computed here from the in-plane velocity components. Note that the real streamlines are helicoidal. The streamlines shown here actually correspond to the in-plane projection of the 3D streamlines (due to the invariance of our problem along the out-of-plane direction). We find that the streamlines closely follow the back of the cylinder up to the critical Reynolds number $\operatorname{Re}_{c}=(275 \pm 25) / \operatorname{Ro}$, whereas in a nonrotating fluid, separation occurs at $\operatorname{Re} \simeq 3-4$, well before the wake instability at $\operatorname{Re}_{c} \simeq 47$. Here, above the critical Reynolds number, the boundary layer detaches and directly becomes unstable.

When crossing the transition line Re Ro $\simeq 275$, the wake actually transits directly from steady to turbulent. The nature of the wake close to the transition line Re Ro $\simeq 275$ is illustrated in Fig. 10 for eight values of $(\mathrm{Re}, \mathrm{Ro})$, four below and four above the transition. The steady wakes, shown in 

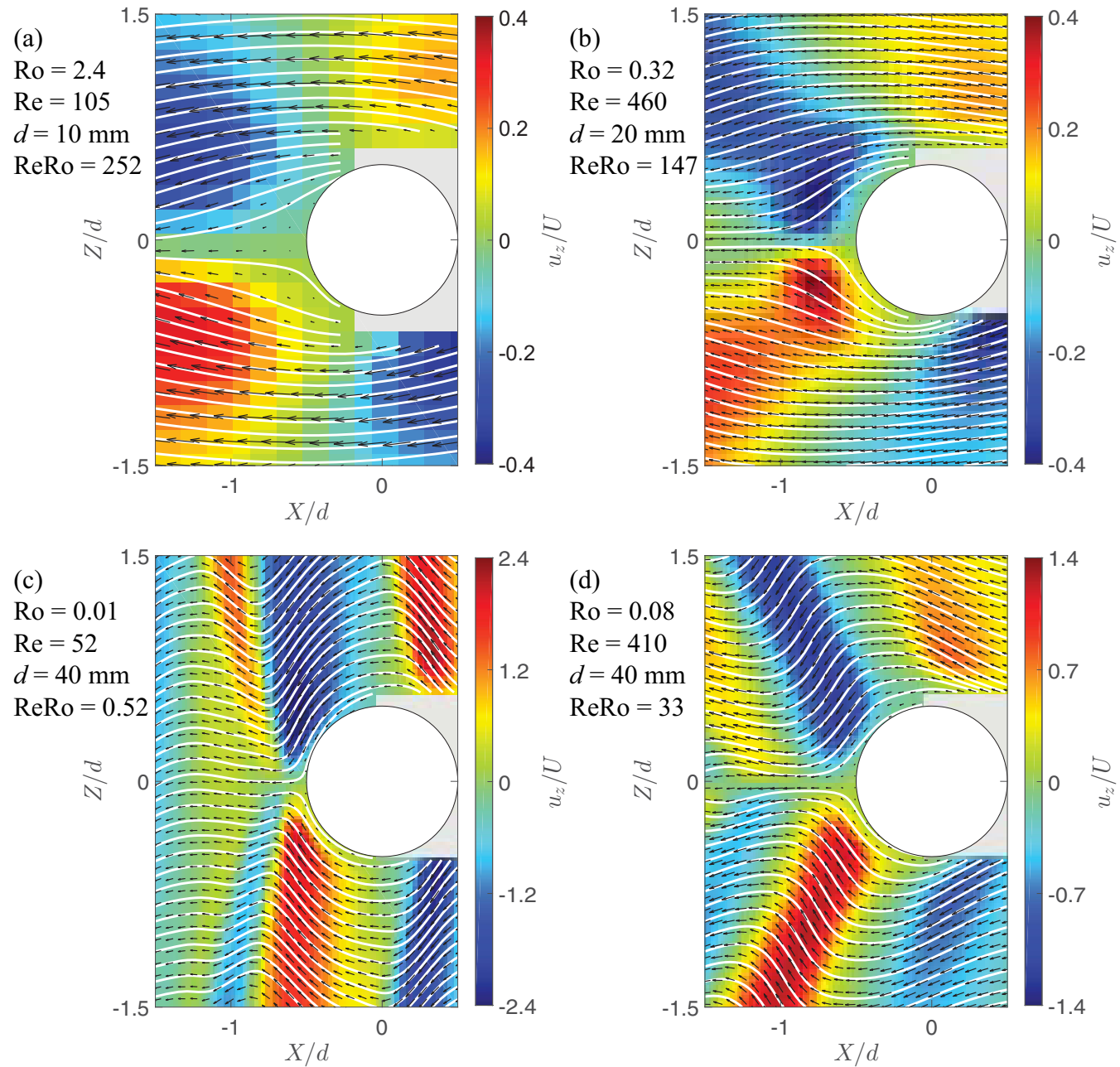

FIG. 9. Close-up view of the experimental wake of a cylinder for (a) $\operatorname{Ro}=2.4, \operatorname{Re}=105$, (b) $\operatorname{Ro}=0.32$, $\operatorname{Re}=460$, (c) $\operatorname{Ro}=0.01, \operatorname{Re}=52$, and (d) $\mathrm{Ro}=0.08, \mathrm{Re}=410$, for which the wake is steady. Each panel reports the time-averaged velocity field in the cylinder reference frame: Arrows show the in-plane velocity components $\left(u_{x}, u_{z}\right)$ and the colormap shows the vertical velocity component $u_{z}$ normalized by $U$. White lines show streamlines of the in-plane velocity field.

Figs. 10(a), 10(c), 10(e), and 10(g), have a structure in good agreement with the predicted lines of constant phase for a line disturbance (41) and (42); systematic comparisons with the model developed in Sec. II B are provided in Sec. III C. The unsteady wakes in Figs. 10(b), 10(d), 10(f), and 10(h) show a combination of turbulent vortices, confined in a $\pm 20^{\circ}$ angular sector centered on the wake axis, and a steady component similar to the steady wake of inertial waves but of weak amplitude. Interestingly, this steady component of the unsteady wakes is still reasonably well described by the predicted phase lines for a line disturbance. It however rapidly vanishes as Re Ro increases, as can be seen in Fig. 11.

At large Rossby number, the unsteady wake resembles a von Kármán vortex street but progressively becomes more and more turbulent as Ro decreases at constant Re (see Fig. 11 for $\mathrm{Re}=840$ and corresponding movies in the Supplemental Material [29]). A regular pattern with periodic vortex shedding such as in Fig. 11(a), typical of a nonrotating von Kármán vortex street, is 

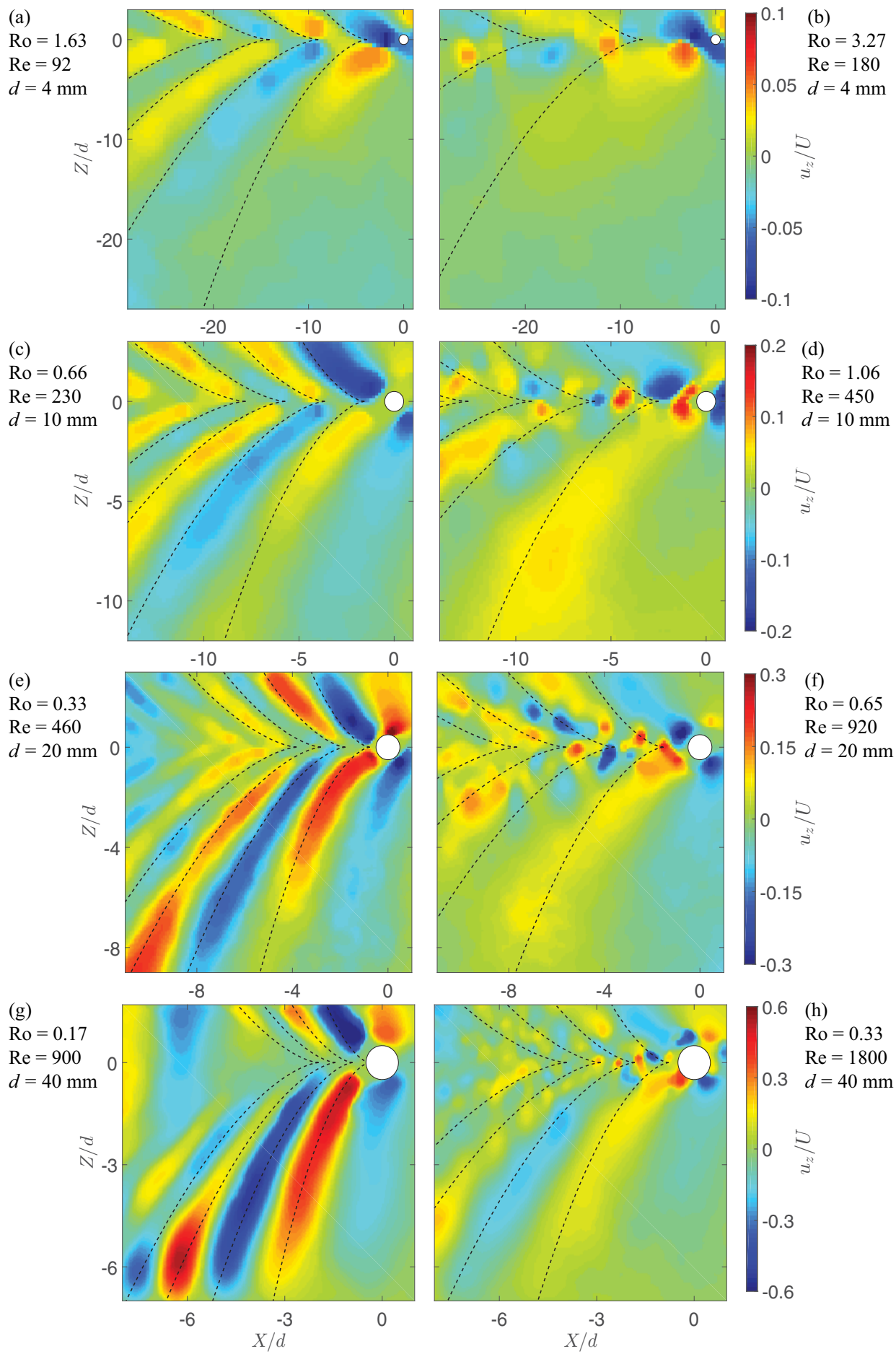

FIG. 10. Instantaneous fields of the vertical velocity component $u_{z}$ normalized by $U$ for several couples (Re, Ro) close to the onset of nonstationarity: (a), (c), (e), and (g) steady wakes and (b), (d), (f), and (h) unsteady wakes. We show as dashed lines theoretical lines of constant phase (41) and (42) of the far-field wake (37)-(39) for $\varphi_{s}+\pi / 2=\pi, 2 \pi, 3 \pi$, and $4 \pi$, from right to left. 


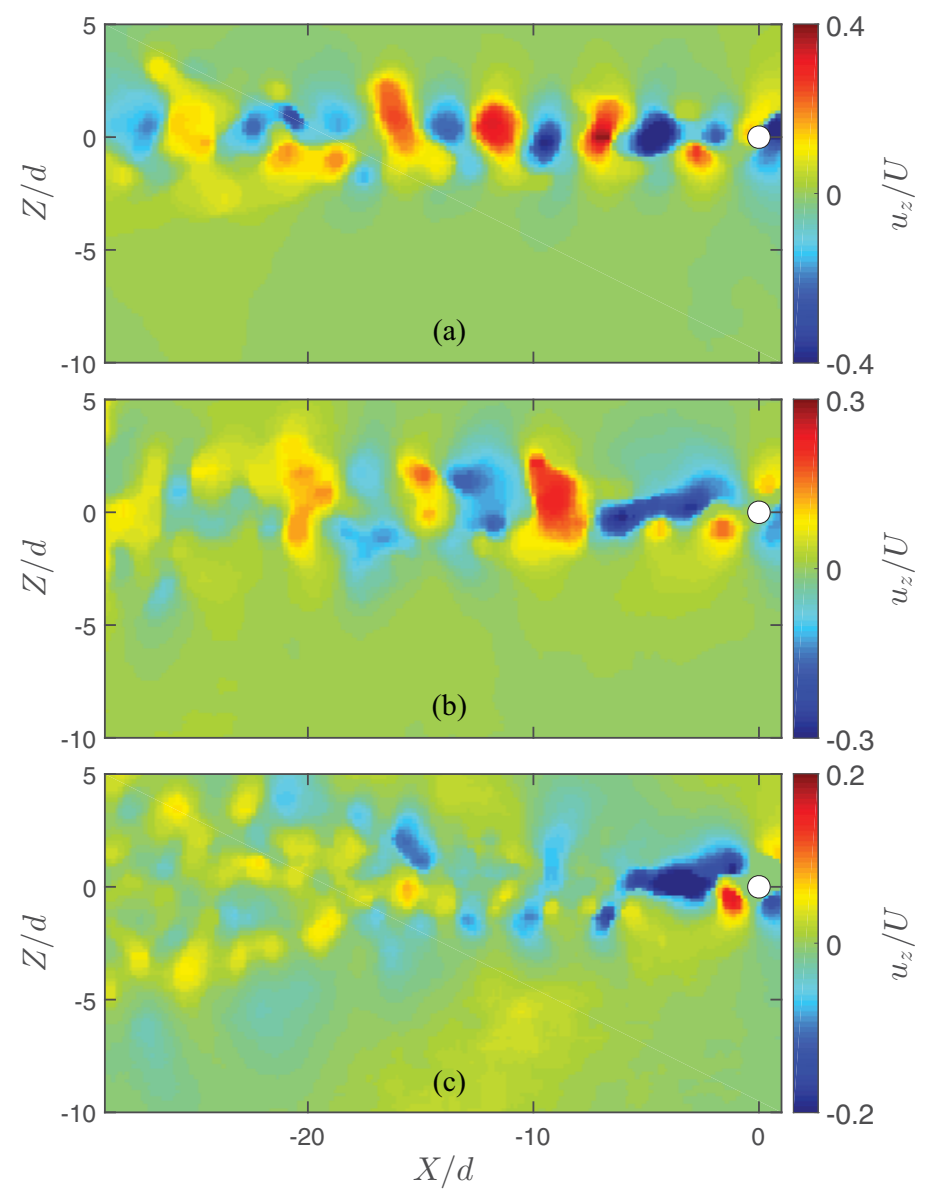

FIG. 11. Instantaneous fields of the vertical velocity component $u_{z}$ normalized by $U$ for a fixed Reynolds number $\operatorname{Re}=840$ and a decreasing Rossby number: (a) Ro $=19.7$, (b) Ro $=4.92$, and (c) Ro $=1.97$. The cylinder diameter is $d=10 \mathrm{~mm}$.

actually observed only for Rossby numbers larger than about 5 (closed markers in Fig. 8). For these experiments, the shedding frequency $f$ yields Strouhal numbers $\mathrm{St}=f d / U$ in the range $0.15-0.20$, in good agreement with typical values found in nonrotating fluids [30].

\section{Steady wake of inertial waves}

In the following we focus on the range of Reynolds and Rossby numbers (Re,Ro) for which the wake is stationary. Snapshots of such wakes are shown in Fig. 12, for increasing Ro in the range $0.01-0.75$, with Reynolds numbers kept nearly constant $(21 \leqslant \mathrm{Re} \leqslant 52)$. Although the wakes are steady, a temporal average in the reference frame of the cylinder is applied to filter out residual unsteady fluid motions which are not related to the wake; these unsteady contributions, of the order of $1 \mathrm{~mm} \mathrm{~s}^{-1}$, mainly originate from residual thermal convection and from a weak precession motion due to the coupling of the platform rotation with the earth rotation [34].

For Rossby numbers typically larger than 0.15 [Figs. 12(d)-12(f)], the structure of the wake is in excellent agreement with the predicted phase lines (41) and (42) for an infinitely small object, shown as dashed lines. As the Rossby number is decreased below 0.15 [Figs. 12(a)-12(c)], the wake pattern is no longer correctly described by these lines of constant phase: The wake becomes more vertically 

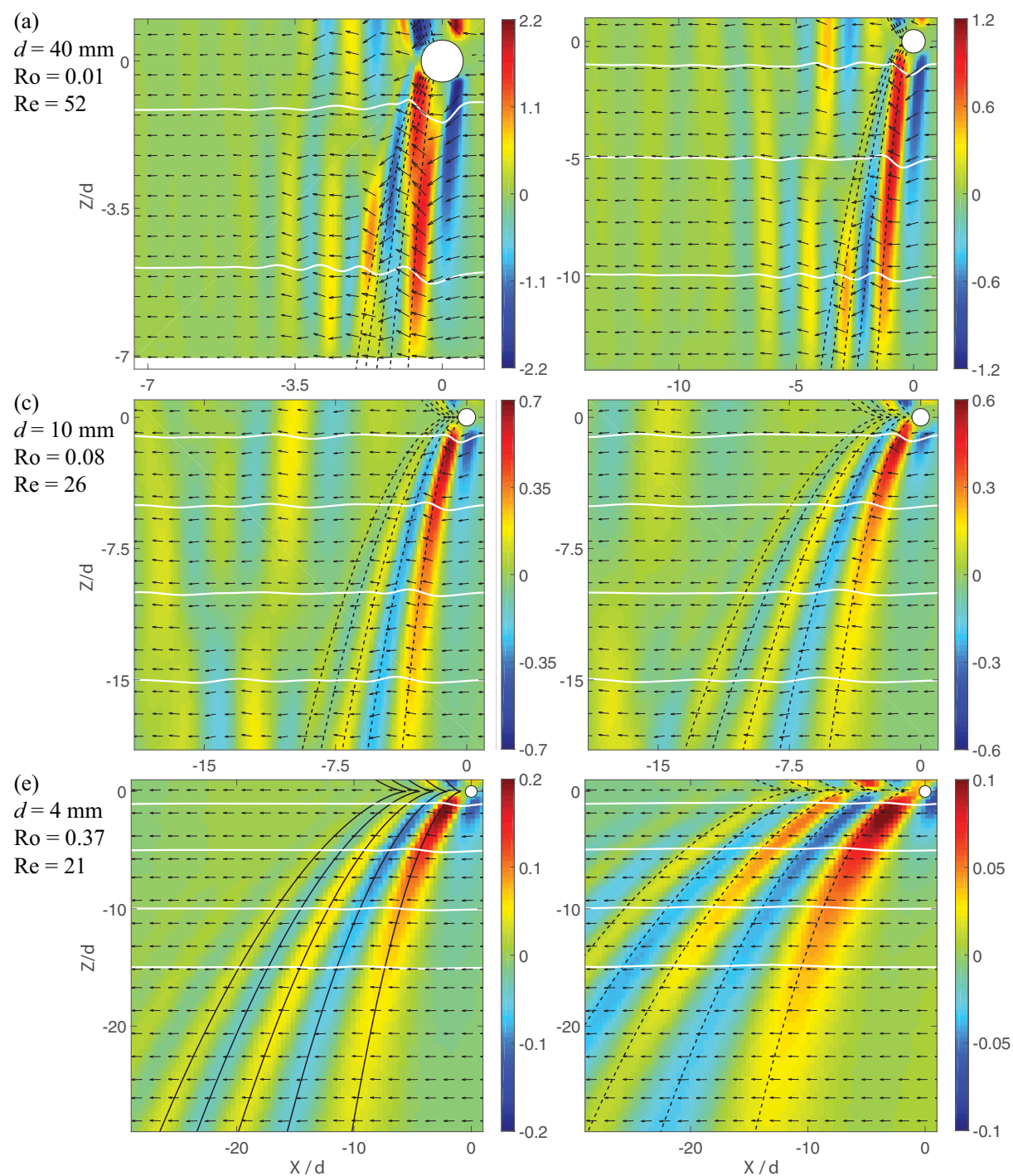
$d=10 \mathrm{~mm}$ $\mathrm{Ro}=0.15$ $\operatorname{Re}=26$

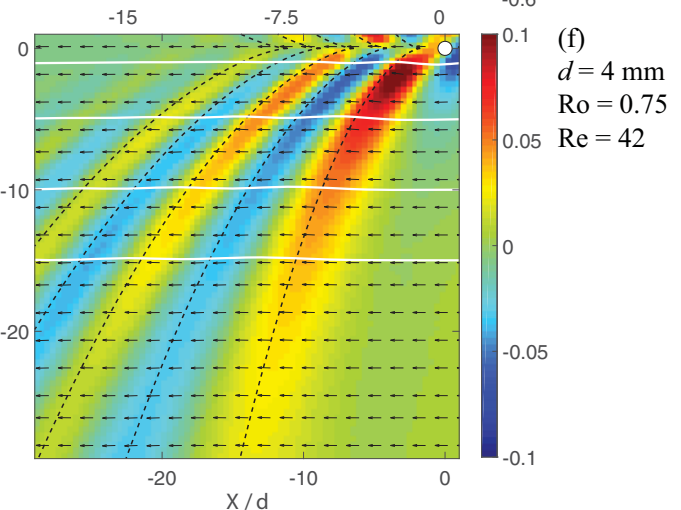

FIG. 12. Experimental wake for increasing Ro and nearly constant $\operatorname{Re}(21 \leqslant \operatorname{Re} \leqslant 52)$. Each panel shows the time-averaged velocity field in the reference frame of the cylinder: Arrows show the in-plane velocity components $\left(u_{x}, u_{z}\right)$ and the colormap shows the vertical velocity component $u_{z}$ normalized by $U$. White lines are streamlines of the in-plane velocity field; assuming the flow invariance along $y$, these lines are the projection in the vertical plane of the real streamlines. Dashed lines are the predicted lines of constant phase (41) and (42) of the far-field wake for $\varphi_{s}+\pi / 2=\pi, 2 \pi, 3 \pi, 4 \pi$, and $5 \pi$.

invariant than predicted by the theory for a line object (note that these snapshots contain additional wave beams that correspond to reflections on the free surface and the bottom of the tank). This discrepancy between the measured wake and the theoretical lines of constant phase (41) and (42), which is larger as Ro is decreased, originates from the growing influence of the size of the cylinder. This is natural since, when Ro $=\lambda_{0} / 2 \pi d$ becomes lower than $\sim 0.15$, the characteristic wavelength along the axis of the theoretical wake of a line object, $\lambda_{0}=\pi U / \Omega$, becomes smaller than the cylinder diameter $d$. This is also in agreement with the theoretical results of Sec. IIB, which have revealed the finite-size effects to become significant below typically Ro $\sim 0.1$. These observations illustrate 

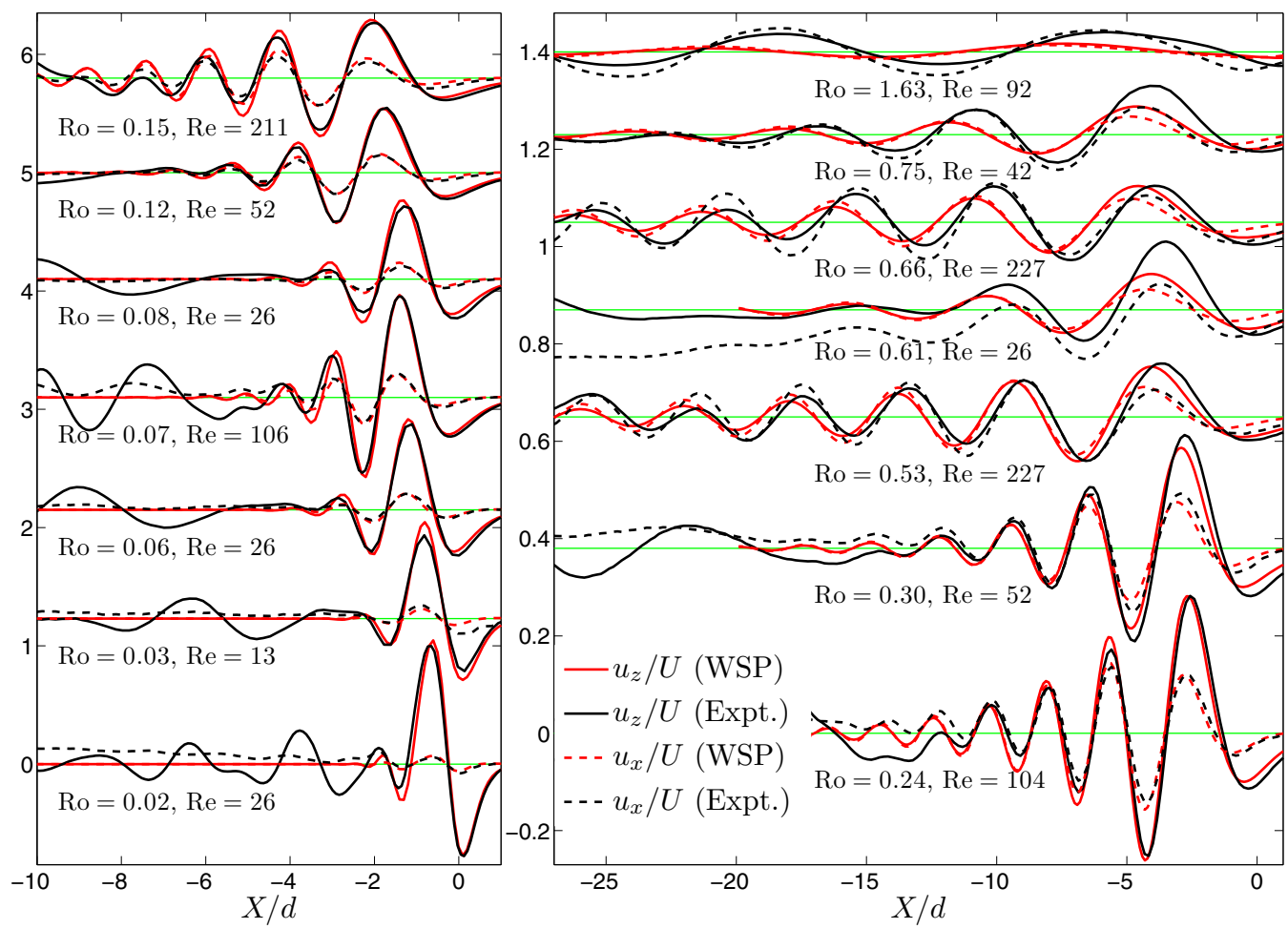

FIG. 13. Normalized axial $u_{x} / U$ (dotted lines) and vertical $u_{z} / U$ (solid lines) velocity profiles at $Z / d=-3$ as a function of $X / d$ for several values of $(\mathrm{Ro}, \mathrm{Re})$ for the experiments (black lines) and for the prediction based on the weak-streamwise-perturbation approximation (red lines, denoted by WSP in the legend).

the need to consider the model (31)-(33) accounting for the shape and finite size of the cylinder to describe the experimental wake at low Ro. This comparison is further provided in Figs. 13 and 14.

This nearly vertically invariant wake observed at low Ro [Figs. 12(a) and 12(b)], of width of the order of the cylinder diameter, is essentially composed of a slice of downward fluid motion below the "bow" of the cylinder followed by a slice of upward fluid motion below the "stern" of the cylinder. Although this increasing vertical invariance is consistent with the Taylor-Proudman theorem, this flow is not a Taylor column, which is prohibited in the case of a 2D object (invariant along $y$ ). In the limit of very small Rossby number, the wake stationarity condition in the frame of the cylinder implies that low-frequency waves compose the wake in the frame of the fluid at rest. Then the only fluid motions allowed by the wave dispersion relation are circular translations in vertical planes. Accordingly, here, in the frame of the fluid at rest, any fluid particle at a given $x$ must describe, during the transit of the cylinder, one circular translation in the vertical plane $(y, z)$, oriented by $\mathbf{e}_{x}$ $\left(\mathbf{e}_{x} \cdot \mathbf{U}>0\right)$ below the object and by $-\mathbf{e}_{x}$ above. Time symmetry of the wave dynamics then implies that the fluid particle must return to its initial position after the transit of the cylinder; this explains why the upstream-downstream nonsymmetric separated wake flow, typical in nonrotating fluids, tends to be inhibited at small Rossby number here, in good agreement with the streamlines reported in Fig. 9.

In order to compare the experiments with the theory of Sec. IIB, i.e., Eqs. (31)-(33), we report in Fig. 13 the measured and predicted axial profiles of the normalized vertical $u_{z} / U$ and axial $u_{x} / U$ velocity components at height $Z / d=-3$ for increasing Rossby number and various Reynolds numbers. In the experimental profiles at Ro $\lesssim 0.1$, the unexpected oscillations behind the cylinder, observed in the region where the theoretical profiles are essentially flat, are due to the reflection of 


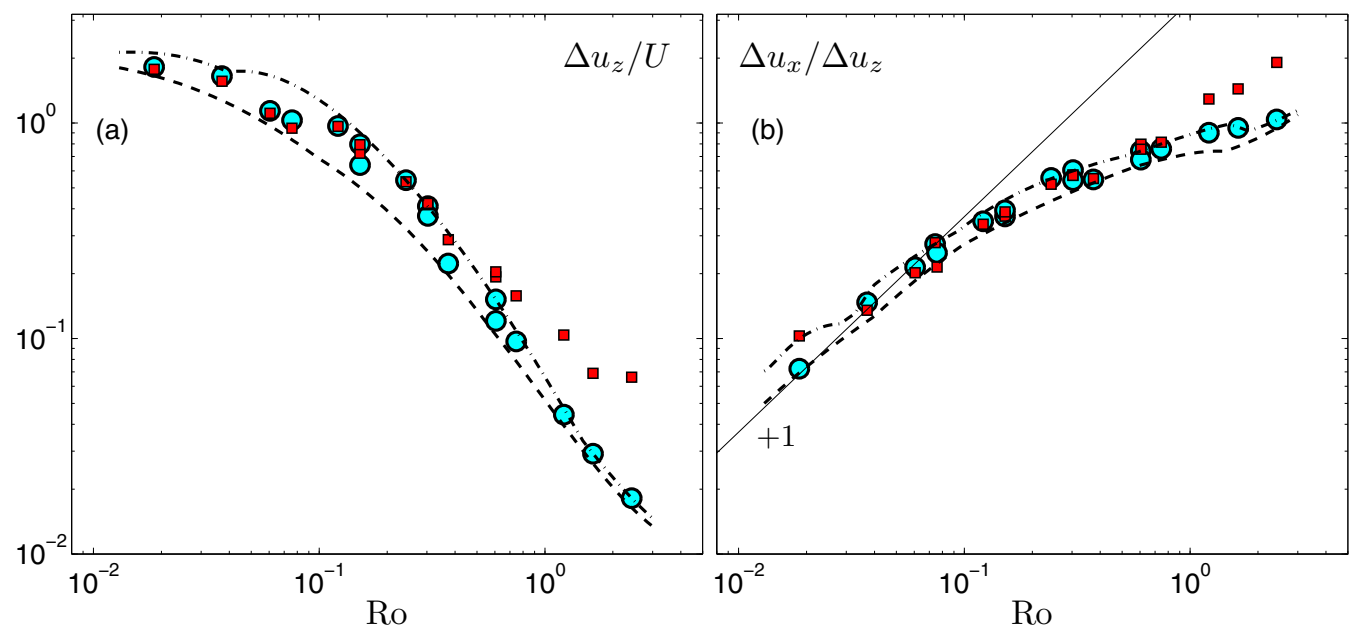

FIG. 14. (a) Amplitude $\Delta u_{z} / U$ of the maximum vertical velocity oscillation at $Z / d=-3$ as a function of Ro for all the experiments with $15 \lesssim \operatorname{Re} \lesssim 100$. For each data point (squares), the prediction based on the weak-streamwise-perturbation approximation for the same $(\mathrm{Ro}, \mathrm{Re})$ values is reported with a circle. (b) Corresponding ratio $\Delta u_{x} / \Delta u_{z}$ of the axial to vertical maximum velocity oscillation at $Z / d=-3$. The straight line shows the expected asymptotic behavior at low Ro, $\Delta u_{x} / \Delta u_{z} \simeq k_{x, \text { extr }}^{1} d$ Ro. In both figures, the dashed and dash-dotted lines show the theoretical predictions of the weak-streamwise-perturbation model for $\operatorname{Re}=15$ and $\operatorname{Re}=100$, respectively.

the wake at the bottom of the water tank and at the fluid free surface. Focusing on the distances $X$ where a nonflat profile is predicted, we observe quantitative agreement between the experimental data and the model for Ro typically lower than 0.30 , i.e., when significant finite-size effects are present. For larger Ro, we note an increasing wake amplitude and upstream phase shift compared to the prediction. Such an upstream phase shift was also found by Heikes and Maxworthy [12] for a ridge made of a portion of a cylinder, but with a weaker wave amplitude. They attributed this weaker wave amplitude to viscous damping, which was not included in their model. In any case, at Rossby numbers similar to those in Ref. [12], we observe quantitative agreement between the model and our experiments.

To provide further comparison with the model, we consider the variation with Ro of the amplitude of the main oscillation behind the cylinder. In Fig. 14(a) we plot the difference $\Delta u_{z} / U$ between the maximum and minimum vertical velocity in the wake at $Z / d=-3$ for all the experiments with $15 \lesssim \operatorname{Re} \lesssim 100$ (this range is chosen in order to limit the dispersion due to viscosity while keeping a significant number of data points). Experimental data are shown with square markers and the corresponding model predictions with circles; the predictions for the two limiting values $\operatorname{Re}=15$ and $\operatorname{Re}=100$ are shown with dashed and dash-dotted lines, respectively. For Ro $\leqslant 0.3$, we observe excellent agreement between theory and experiment, while for Ro $>0.3$, the measured wake amplitude becomes larger than the prediction, up to a factor 4 for Ro $=3$. We cannot test the evolution of the discrepancy between the steady wake theory and the experiments at Ro $>3$ because it corresponds here to the largest Rossby number at which a stable wake is observed (see Fig. 8).

We finally show in Fig. 14(b) the ratio $\Delta u_{x} / \Delta u_{z}$ of the axial to vertical maximum velocity oscillations at $Z / d=-3$ for the experiments and the model as a function of Ro. We observe good agreement between the experiment and the model up to Ro $\simeq 0.8$. In addition, one can note that the linear behavior observed at very small Ro simply follows from the stationarity condition (10), yielding $\Delta u_{x} / \Delta u_{z}=U k / 2 \Omega$, where $k$ is the local wave number. Indeed, at small Rossby numbers, when finite-size effects are important, the dominant wave number in the wake scales as $k \sim k_{x} \sim$ $2 \pi / d$. Using the first extrema of the cylinder spectrum (51) to estimate this dominant wave number, 
we recover the observed linear behavior $\Delta u_{x} / \Delta u_{z} \simeq k_{x, \text { extr }}^{1} d$ Ro $\simeq 3.68$ Ro. We finally note that, for Ro $\lesssim 0.30$ for which the model describes quantitatively the observed wakes, the velocity ratio $\Delta u_{x} / 2 U$ remains below 0.2 , which provides a justification for the approximation of weak streamwise perturbation used in the model.

At moderate and large Rossby numbers, the choice of the model for the translating object becomes unimportant for the global phase pattern, which matches the line object model as shown, for example, in Figs. 12(e) and 12(f). Nevertheless, understanding the flow close to the object remains decisive in describing the wake amplitude and phase origin (Figs. 13 and 14). In this context, it is clear that for the moderate Rossby numbers in the range $0.3 \lesssim$ Ro $\lesssim 3$, the weak-streamwise-perturbation boundary condition does not describe well the actual boundary condition for the wave field, calling for a more accurate description of the viscous boundary layer on the cylinder.

\section{CONCLUSION}

In this article we have studied experimentally the wake produced by the horizontal translation at constant velocity of a horizontal cylinder in a fluid rotating about the vertical axis. For steady wakes, we proposed a model of wake of inertial waves based on an earlier model by Johnson [3] that retains the weak-streamwise-perturbation and infinite-depth approximations of Johnson but relaxes the quasigeostrophic approximation. We showed that this model describes the experiments quantitatively for Ro $\lesssim 0.3$ and for Re ranging from 1 to $10^{3}$. Our measurements confirm that this approximation of a weak streamwise perturbation applied in a free-slip boundary condition leads to an accurate description of the wake even for a nonslender object, provided it is horizontally invariant in the cross-stream direction. This result follows from the fact that the vertical-to-horizontal velocity ratio is imposed by the frequency in an inertial wave. Here we showed that this low-Ro prediction applies even at moderate Rossby number. Our experimental validation of the weak-streamwiseperturbation model at low Ro opens the way for a theoretical exploration of the influence on the wake of inertial waves of the object's shape for arbitrary horizontal 2D bluff body.

At large Rossby numbers, the structure of the wake is expected to become independent of the size of the object, and we indeed recovered experimentally the wake predicted for a line object by Lighthill [8] for Ro larger than 0.3. However, our measurements show that the weak-streamwise-perturbation approximation no longer allows one to predict the correct amplitude and phase origin of the wake in this regime. These results call for a better description of the viscous boundary layers along the cylinder at moderate Rossby number.

In this work we also studied the threshold in Reynolds and Rossby numbers above which the wake becomes unsteady. We found a strong stabilization by rotation of the wake, which remains steady up to $\operatorname{Re}_{c} \sim 1000$ at $\operatorname{Ro} \sim 0.3$, to be compared with the threshold for the appearance of the von Kármán vortex street, $\operatorname{Re}_{c} \sim 50$, in a nonrotating fluid. Close-up views behind the cylinder indicate that in this stabilized regime the boundary layer detachment is inhibited by rotation. The stability criterion suggested by our experiments, $\operatorname{Re}<(275 \pm 25) /$ Ro, indicates a competition between the inertial and Ekman time scales, but no general stability analysis for this problem is available yet.

All these results are for two-dimensional objects, invariant along the cross-stream horizontal direction. For three-dimensional objects, the prediction of the full velocity field of the wake of inertial waves implies the identification of a relevant description of the boundary layers on the object, which is still an open question in the general case. Indeed, the weak-streamwise-perturbation approximation requires the object to be slender and is no longer valid for 3D bluff bodies. It has been applied to the calculation of the waves by Cheng and Johnson [11] and compared with experiments involving spherical caps and vertical pillars by Heikes and Maxworthy [12], who pointed out, in particular, a lateral deflection that may be caused by the geostrophic flow around the object. The same structure is also visible in the numerical simulations of Mason and Sykes [6]. One should remember this second difference with the 2D case treated in this article: As Ro decreases, a Taylor-column vertically invariant flow $[4,7]$ is expected to appear and finally dominate the wake in fluid domains of finite depth. 
In geophysical configurations, the stratification of the fluid, characterized by the Brunt-Väisälä frequency $N$, plays a significant role in addition to rotation. The problem becomes that of the horizontal flow at velocity $U$ over a topography of streamwise size $L$, with the internal Froude number $\mathrm{Fr}=U / N L$ a new parameter. Inertia-gravity waves are generated, composed of transverse and converging waves contained by a caustic, the streamwise orientation of the transverse and converging waves being reversed depending on whether $N$ is smaller or larger than $2 \Omega$ [9]. The weak-streamwise-perturbation approximation has been applied to this configuration by Cheng et al. [35] for thin topographies.

Geophysical flows usually have low internal Froude numbers, corresponding to strong stratification, in association with moderately low Rossby numbers. When only stratification is present, vertical motion is confined to a small layer of height $U / N=L$ Fr below the top of the topography, while the fluid below that layer flows horizontally around the topography. The horizontal surface separating the two layers is called the dividing streamline, or more exactly a stream surface. Since vertical motion is the primary cause of internal wave motion, only the portion of the topography protruding above the dividing stream surface contributes to the wave radiation, acting as a thin cutoff obstacle to which the weak-streamwise-perturbation approximation may be applied. This approach was evoked by Newley et al. [36], Hunt et al. [37], and Greenslade [38] and then implemented by Hunt et al. [39], Voisin [17], and Dalziel et al. [40], the latter also presenting experiments for hemispherical topography. As a result, the weak-streamwise-perturbation approximation turns out to also be applicable to three-dimensional bluff topography in a stratified flow, so long as the stratification is strong. Generalization to a rotating and stratified fluid depends on the deformation that rotation may impose on the dividing stream surface, a topic that deserves further investigation.

\section{ACKNOWLEDGMENTS}

We acknowledge M. Rabaud, S. Le Dizès, and E. R. Johnson for fruitful discussions and J. Amarni, A. Aubertin, L. Auffray, and R. Pidoux for experimental help. This work is supported by "Investissements d'Avenir" LabEx PALM (ANR-10-LABX-0039-PALM).

\section{APPENDIX A: FAR-FIELD EXPANSION OF WEAKLY DISSIPATIVE WAVES}

We look for the expansion of integrals of the form

$$
I(\lambda)=\int_{a}^{b} g(k) e^{i \lambda f(k)} d k
$$

with $a$ and $b$ real, as $\lambda \rightarrow \infty$, in the particular case where

$$
f(k)=f_{0}(k)+i \epsilon f_{1}(k),
$$

with $f_{0}(k)$ and $f_{1}(k)$ real and $\epsilon \ll 1$. Such integrals are met when investigating the effect of weak dissipation on the propagation of waves in the far field, as in [3] and Sec. IIB. We assume that $g(k)$ is regular on the interval of integration and $f_{0}(k)$ has a simple stationary point $k_{0}$ such that $f_{0}^{\prime}\left(k_{0}\right)=0$ and $f_{0}^{\prime \prime}\left(k_{0}\right) \neq 0$. For $\epsilon=0$, the method of stationary phase (see [41], Sec. 2.7) yields

$$
I(\lambda) \sim \sqrt{\frac{2 \pi}{\lambda\left|f_{0}^{\prime \prime}\left(k_{0}\right)\right|}} g\left(k_{0}\right) \exp \left[i \lambda f_{0}\left(k_{0}\right)+i \frac{\pi}{4} \operatorname{sgn} f_{0}^{\prime \prime}\left(k_{0}\right)\right] .
$$

When $\epsilon \neq 0$, to $O(\epsilon)$, the real stationary point becomes a complex saddle point

$$
k_{s} \sim k_{0}-i \epsilon \frac{f_{1}^{\prime}\left(k_{0}\right)}{f_{0}^{\prime \prime}\left(k_{0}\right)}
$$


such that $f^{\prime}\left(k_{s}\right)=0$ and at which

$$
\begin{gathered}
f\left(k_{s}\right) \sim f_{0}\left(k_{0}\right)+i \epsilon f_{1}\left(k_{0}\right) \sim f\left(k_{0}\right), \\
f^{\prime \prime}\left(k_{s}\right) \sim f_{0}^{\prime \prime}\left(k_{0}\right)+i \epsilon\left[f_{1}^{\prime \prime}\left(k_{0}\right)-f_{1}^{\prime}\left(k_{0}\right) \frac{f_{0}^{\prime \prime \prime}\left(k_{0}\right)}{f_{0}^{\prime \prime}\left(k_{0}\right)}\right] \sim f^{\prime \prime}\left(k_{0}\right)-i \epsilon f_{1}^{\prime}\left(k_{0}\right) \frac{f_{0}^{\prime \prime \prime}\left(k_{0}\right)}{f_{0}^{\prime \prime}\left(k_{0}\right)} .
\end{gathered}
$$

The steepest-descent path through this point, oriented towards increasing $\mathfrak{R e}(k)$, makes an angle $\theta_{p}=\pi / 2-\arg \left[i f^{\prime \prime}\left(k_{s}\right)\right] / 2(\bmod \pi)$ with the $\mathfrak{R e}(k)$ axis, that is,

$$
\theta_{p} \sim \frac{\pi}{4} \operatorname{sgn} f_{0}^{\prime \prime}\left(k_{0}\right)-\epsilon \frac{f_{1}^{\prime \prime}\left(k_{0}\right) f_{0}^{\prime \prime}\left(k_{0}\right)-f_{1}^{\prime}\left(k_{0}\right) f_{0}^{\prime \prime \prime}\left(k_{0}\right)}{2\left[f_{0}^{\prime \prime}\left(k_{0}\right)\right]^{2}} .
$$

The method of steepest descent (see [41], Chap. 7) yields

$$
\begin{aligned}
I(\lambda) \sim & \sqrt{\frac{2 \pi}{\lambda\left|f_{0}^{\prime \prime}\left(k_{0}\right)\right|}} g\left(k_{0}\right) \exp \left[-\epsilon \lambda f_{1}\left(k_{0}\right)\right] \\
& \times \exp \left\{i \lambda f_{0}\left(k_{0}\right)+i \frac{\pi}{4} \operatorname{sgn} f_{0}^{\prime \prime}\left(k_{0}\right)-i \epsilon \frac{f_{1}^{\prime \prime}\left(k_{0}\right) f_{0}^{\prime \prime}\left(k_{0}\right)-f_{1}^{\prime}\left(k_{0}\right) f_{0}^{\prime \prime \prime}\left(k_{0}\right)}{2\left[f_{0}^{\prime \prime}\left(k_{0}\right)\right]^{2}}\right\} .
\end{aligned}
$$

Two contributions are added to the phase $\lambda f_{0}\left(k_{0}\right)$ : one $O(\epsilon \lambda)$, affecting the amplitude of the waves and which, depending on $\lambda$ and $\epsilon$, may be $O(1)$ and hence must be retained, and the other $O(\epsilon)$, affecting the phase of the waves and expected to be negligible. Accordingly, the dominant effect of small nonzero $\epsilon$ is obtained by evaluating the disturbed $f(k)$ at the undisturbed stationary point $k_{0}$, following (A5).

\section{APPENDIX B: VISCOUS DEFORMATION OF THE LINES OF CONSTANT PHASE}

Quasigeostrophy allowed Johnson [3] to consider viscosity at any Reynolds number Re. In the far field, the steepest-descent method not only confirmed the exponential amplitude attenuation expected from the group-velocity theory but also revealed a viscous deformation of the lines of constant phase, which starts to be significant, relative to $\lambda_{0}$, the characteristic wavelength of the inviscid wake, at Re Ro of order unity and makes the lines straighter near the origin. Here, without quasigeostrophy, an assumption of large $\operatorname{Re}_{k_{0}}=U / \nu k_{0}=\operatorname{Re} \operatorname{Ro}$ had to be introduced to keep the analysis tractable. Appendix A, with $\epsilon=1 /(\operatorname{Re} R o)$, then predicted the deformation to be negligible. In the following, we consider this deformation nonetheless, in order to assess its connection with the work of Johnson [3].

The phase (40) becomes

$$
\varphi_{s}=k_{0}|Z| \frac{\cos ^{2} \theta}{\sin ^{3} \theta}-\frac{\pi}{4}+\frac{1}{\operatorname{Re} \operatorname{Ro}} \frac{\cos \theta}{\sin ^{4} \theta} \frac{6+8 \cos ^{2} \theta+\cos ^{4} \theta}{4+4 \cos ^{2} \theta+\cos ^{4} \theta},
$$

yielding, for the lines of constant phase, the parametric equations

$$
\begin{gathered}
k_{0}|X|=\frac{1+\sin ^{2} \theta}{\cos \theta}\left(\varphi_{s}+\frac{\pi}{4}-\frac{1}{\operatorname{Re} \operatorname{Ro}} \frac{\cos \theta}{\sin ^{4} \theta} \frac{6+8 \cos ^{2} \theta+\cos ^{4} \theta}{4+4 \cos ^{2} \theta+\cos ^{4} \theta}\right), \\
k_{0}|Z|=\frac{\sin ^{3} \theta}{\cos ^{2} \theta}\left(\varphi_{s}+\frac{\pi}{4}-\frac{1}{\operatorname{Re} \operatorname{Ro}} \frac{\cos \theta}{\sin ^{4} \theta} \frac{6+8 \cos ^{2} \theta+\cos ^{4} \theta}{4+4 \cos ^{2} \theta+\cos ^{4} \theta}\right) .
\end{gathered}
$$

The product Re Ro is seen to govern the importance of the deformation. In Fig. 15 we show the lines of constant phase $\varphi_{s}+\pi / 2=(2 n+1) \pi$ (for $n \in[0 ; 10]$ ) for the three values $\operatorname{Re} \operatorname{Ro}=1,10,100$ and compare them with their inviscid counterpart $\varphi_{s}(\operatorname{Re} \operatorname{Ro}=\infty)+\pi / 2 \equiv \varphi_{0}+\pi / 4=(2 n+1) \pi$. 
(a)

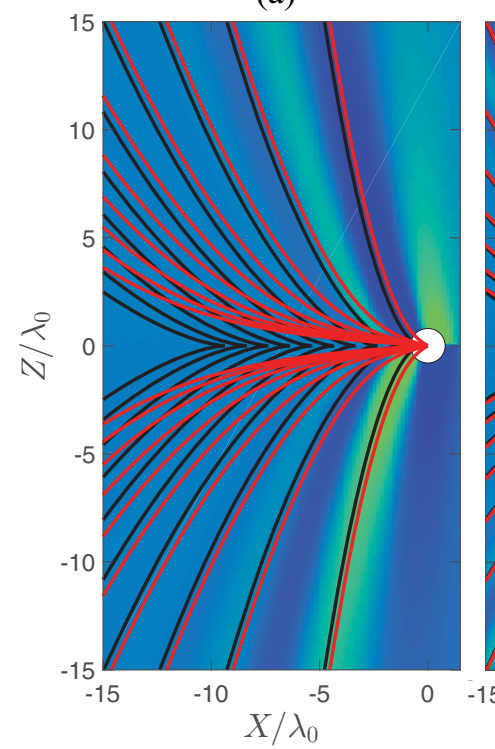

(b)

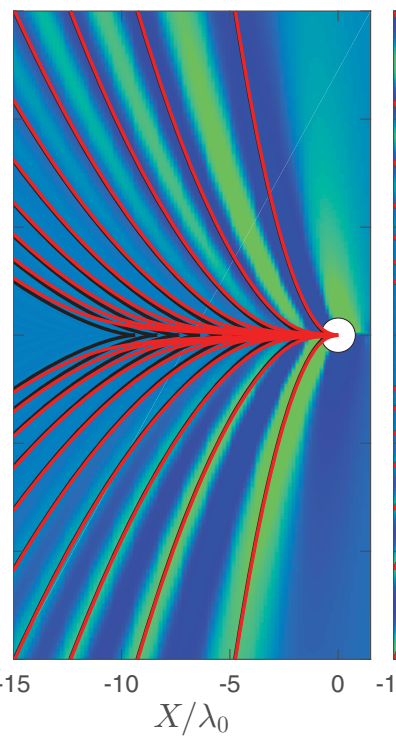

(c)

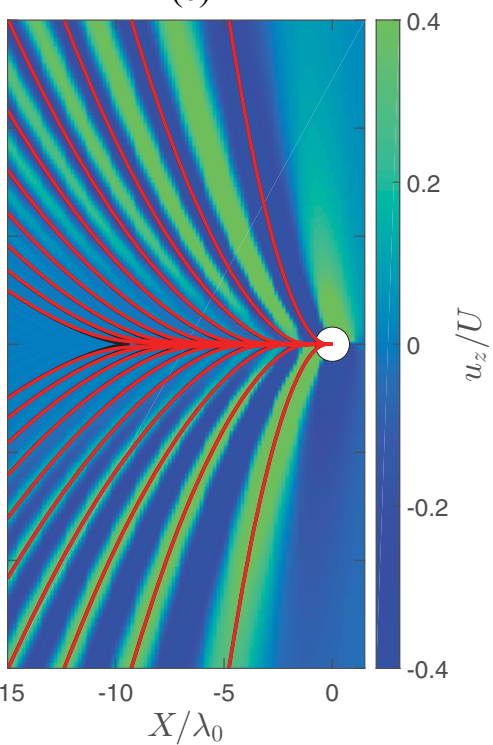

FIG. 15. Lines of constant phase, computed from the viscous model (B2) and (B3) [red lines, for $\varphi_{s}+\pi / 2=$ $(2 n+1) \pi$ and for the three values (a) $\operatorname{Re} \operatorname{Ro}=1$, (b) $\operatorname{Re} \operatorname{Ro}=10$, and (c) $\operatorname{Re} \operatorname{Ro}=100]$ and its inviscid limit (41) and (42) [black lines, for $\varphi_{s}+\pi / 2=(2 n+1) \pi$ and for $\operatorname{Re} \operatorname{Ro}=\infty$ in which case $\varphi_{s}=\varphi_{0}-\pi / 4$ ], for $n \in[0 ; 10]$. The background color shows the vertical velocity of the corresponding prediction by the weakstreamwise-perturbation model of Sec. II B 2 for a cylinder of diameter $d$ and Rossby number Ro $=U / 2 \Omega d=$ 0.1 .

The background color shows the vertical velocity of the corresponding prediction by the weakstreamwise-perturbation model of Sec. II B 2 for a cylinder of diameter $d$ and for Rossby number $\operatorname{Ro}=U / 2 \Omega d=0.1$.

Far from the wake axis $Z=0$, as $\alpha \rightarrow \pi / 2$, the phase reduces to

$$
\varphi_{s}+\frac{\pi}{4} \sim k_{0} \frac{X^{2}}{4|Z|}+\frac{3}{4} \frac{|X| /|Z|}{\operatorname{Re} \operatorname{Ro}}
$$

and the lines of constant phase, of equation

$$
\left(4 \varphi_{s}+\pi\right)|Z| \sim|X|\left(k_{0}|X|+\frac{3}{\operatorname{Re} \operatorname{Ro}}\right)
$$

reduce to the straightened parabolas derived by Johnson [3], corrected for minor typographical errors. Close to the wake axis, as $\alpha \rightarrow 0$, the phase becomes

$$
\varphi_{s}+\frac{\pi}{4} \sim k_{0}|X|-\frac{3}{2} k_{0}|X|^{1 / 3}|Z|^{2 / 3}+\frac{5}{3} \frac{(|X| /|Z|)^{4 / 3}}{\operatorname{Re} \operatorname{Ro}}
$$

and the lines of constant phase, whose inviscid form exhibits evenly spaced cusps on the axis at $k_{0}|X|=\varphi_{s}+\pi / 4$, are stretched into curves with a common corner point at the origin. As expected, the lines of constant phase from the inviscid and viscous models tend to coincide in the limit Re Ro $\rightarrow$ $\infty$. At smaller Re Ro, such that the inviscid and viscous lines differ significantly, the FFT evaluation of the vertical velocity predicted by the weak-streamwise-perturbation model (33) is shown in Fig. 15 to better coincide with the inviscid lines, thereby showing the viscous correction of the lines of constant phase to be of purely rhetorical interest. 


\section{APPENDIX C: UNIFORM FAR-FIELD EXPANSION AT THE WAVE FRONT}

The far-field expansion (37)-(39), in which the large parameter $k_{0}|\mathbf{X}|$ is multiplied by $\cos \theta$, is nonuniform at the wave front $X=0$ where $\theta=\pi / 2$. Mathematically, for the integrals (31)-(33), the nonuniformity is associated with the coalescence of the stationary points $k_{x}= \pm k_{0} \cos \theta$ with the singularity $k_{x}=0$, at which the phase of the integrand is not analytic owing to the presence of $\left|k_{x}\right|$ in (27) and (28). Changing variables to turn the integrals into semi-infinite ones, over $k_{x}>0$, the problem reduces to the coalescence of a stationary point with an end point. Applying the analysis of Bleistein [42], we obtain the uniform expansion

$$
\begin{aligned}
u_{x}=- & \frac{\sin ^{3 / 2} \theta \cos \theta}{\sqrt{2+\cos ^{2} \theta}} \exp \left(-\frac{k_{0}|Z|}{\operatorname{Re} \operatorname{Ro}} \frac{\cos ^{3} \theta}{\sin ^{5} \theta}\right) \frac{1}{2 \sqrt{\lambda_{0}|Z|}} \mathfrak{R e}\left(\hat{q}_{0}\left(\mathbf{k}_{s}\right)\right. \\
& \left.\times\left\{\left[1-(1-i)(C+i S)\left(\sqrt{\frac{2}{\pi} \varphi_{0}}\right) \operatorname{sgn} X\right] e^{-i \varphi_{s}}-i \frac{\operatorname{sgn} X}{\sqrt{\pi \varphi_{0}}}\right\}\right), \\
u_{y}= & -\frac{\sin ^{3 / 2} \theta}{\sqrt{2+\cos ^{2} \theta}} \exp \left(-\frac{k_{0}|Z|}{\operatorname{Re} \operatorname{Ro}} \frac{\cos ^{3} \theta}{\sin ^{5} \theta}\right) \frac{1}{2 \sqrt{\lambda_{0}|Z|}} \mathfrak{I m}\left(\hat{q}_{0}\left(\mathbf{k}_{s}\right)\right. \\
& \left.\times\left\{\left[1-(1-i)(C+i S)\left(\sqrt{\frac{2}{\pi} \varphi_{0}}\right) \operatorname{sgn} X\right] e^{-i \varphi_{s}}-i \frac{\operatorname{sgn} X}{\sqrt{\pi \varphi_{0}}}\right\}\right),
\end{aligned}
$$
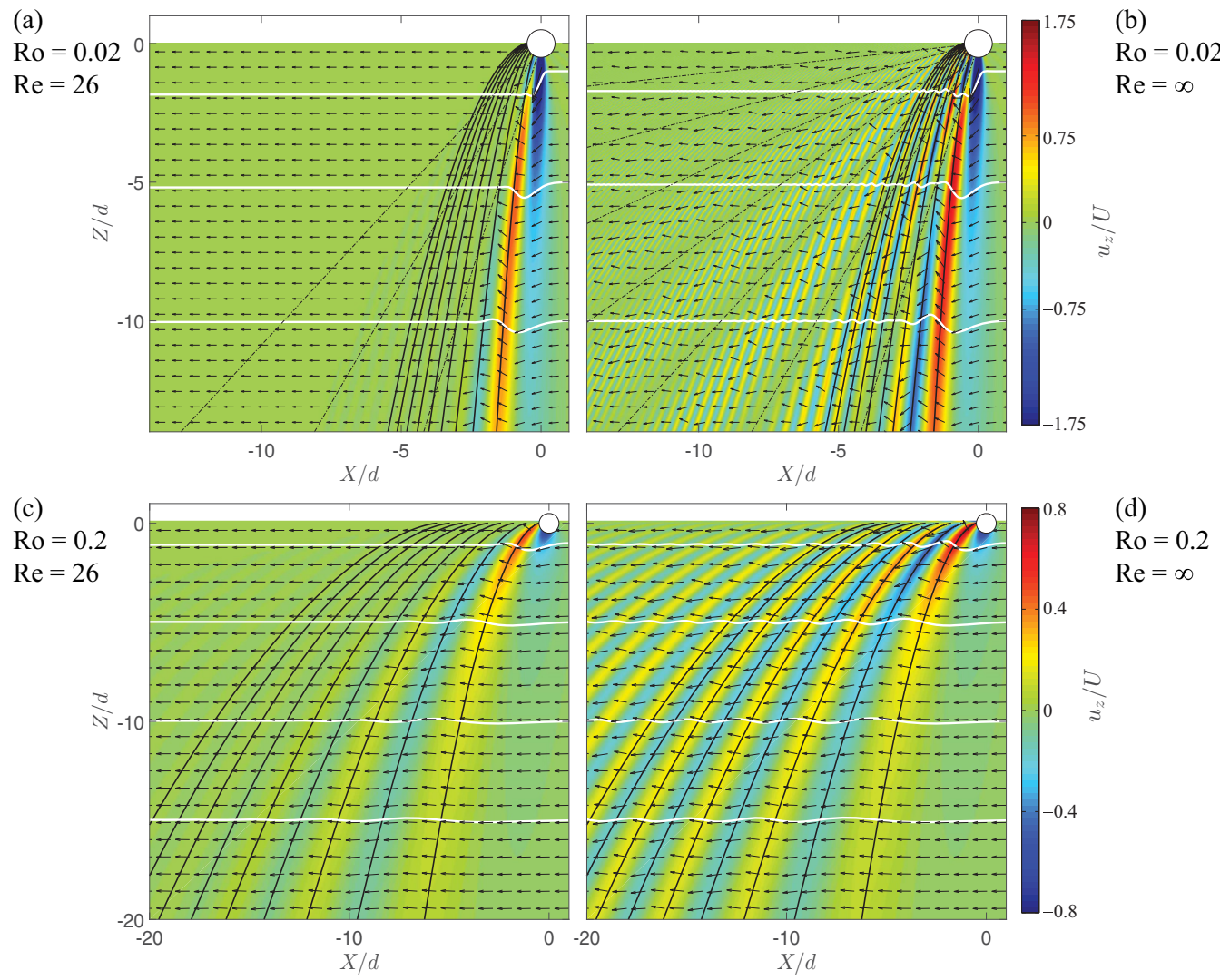

FIG. 16. Wake structure predicted in the uniform far-field approximation $(\mathrm{C} 1)-(\mathrm{C} 3)$, with the same parameters and layout as in Figs. 5 and 6. 


$$
\begin{aligned}
u_{z}= & \frac{\sin ^{5 / 2} \theta \operatorname{sgn} Z}{\sqrt{2+\cos ^{2} \theta}} \exp \left(-\frac{k_{0}|Z|}{\operatorname{Re} \operatorname{Ro}} \frac{\cos ^{3} \theta}{\sin ^{5} \theta}\right) \frac{1}{2 \sqrt{\lambda_{0}|Z|}} \mathfrak{R e}\left(\hat{q}_{0}\left(\mathbf{k}_{s}\right)\right. \\
& \left.\times\left\{\left[1-(1-i)(C+i S)\left(\sqrt{\frac{2}{\pi} \varphi_{0}}\right) \operatorname{sgn} X\right] e^{-i \varphi_{s}}-i \frac{\operatorname{sgn} X}{\sqrt{\pi \varphi_{0}}}\right\}\right),
\end{aligned}
$$

with $C$ and $S$ the Fresnel functions (the term $\sqrt{2 \varphi_{0} / \pi}$ within parentheses is the argument of $C$ and $S$ ). The angles $\alpha$ and $\theta$ vary now between 0 and $\pi$ but remain otherwise linked by (35). The modifications associated with Fig. 6 are represented in Fig. 16.

[1] G. I. Taylor, Experiments on the motion of solid bodies in rotating fluids, Proc. R. Soc. London Ser. A 104, 213 (1923).

[2] H. Greenspan, The Theory of Rotating Fluids (Cambridge University Press, Cambridge, 1968).

[3] E. R. Johnson, The effects of obstacle shape and viscosity in deep rotating flow over finite-height topography, J. Fluid Mech. 120, 359 (1982).

[4] R. Hide and A. Ibbetson, An experimental study of "Taylor columns", Icarus 5, 279 (1966).

[5] R. Hide, A. Ibbetson, and M. J. Lighthill, On slow transverse flow past obstacles in a rapidly rotating fluid, J. Fluid Mech. 32, 251 (1968).

[6] P. J. Mason and R. I. Sykes, A numerical study of rapidly rotating flow over surface-mounted obstacles, J. Fluid Mech. 111, 175 (1981).

[7] K. Stewartson and H. K. Cheng, On the structure of inertial waves produced by an obstacle in a deep, rotating container, J. Fluid Mech. 91, 415 (1979).

[8] M. J. Lighthill, On waves generated in dispersive systems by travelling forcing effects, with applications to the dynamics of rotating fluids, J. Fluid Mech. 27, 725 (1967).

[9] L. G. Redekopp, Wave patterns generated by disturbances travelling horizontally in rotating stratified fluids, Geophys. Fluid Dyn. 6, 289 (1975).

[10] K. S. Peat and T. N. Stevenson, The phase configuration of waves around a body moving in a rotating stratified fluid, J. Fluid Mech. 75, 647 (1976).

[11] H. K. Cheng and E. R. Johnson, Inertial waves above an obstacle in an unbounded, rapidly rotating fluid, Proc. R. Soc. London Ser. A 383, 71 (1982).

[12] K. E. Heikes and T. Maxworthy, Observations of inertial waves in a homogeneous rotating fluid, J. Fluid Mech. 125, 319 (1982).

[13] J. Lighthill, Waves in Fluids (Cambridge University Press, Cambridge, 1978).

[14] O. Darrigol, Words of Flow: A History of Hydrodynamics from the Bernoullis to Prandtl (Oxford University Press, Oxford, 2005).

[15] J. W. Miles, Internal waves generated by a horizontally moving source, Geophys. Fluid Dyn. 2, 63 (1971).

[16] G. S. Janowitz, Lee waves in three-dimensional stratified flow, J. Fluid Mech. 148, 97 (1984).

[17] B. Voisin, Lee waves from a sphere in a stratified flow, J. Fluid Mech. 574, 273 (2007).

[18] F. Waleffe, Inertial transfers in the helical decomposition, Phys. Fluids A 5, 677 (1993).

[19] G. Bordes, F. Moisy, T. Dauxois, and P.-P. Cortet, Experimental evidence of a triadic resonance of plane inertial waves in a rotating fluid, Phys. Fluids 24, 014105 (2012)

[20] W. Thomson (Lord Kelvin), On ship waves, Proc. Inst. Mech. Eng. 38, 409 (1887).

[21] T. H. Havelock, The propagation of groups of waves in dispersive media, with application to waves on water produced by a travelling disturbance, Proc. R. Soc. London Ser. A 81, 398 (1908).

[22] M. Rabaud and F. Moisy, Ship Wakes: Kelvin or Mach Angle?, Phys. Rev. Lett. 110, 214503 (2013).

[23] A. Darmon, M. Benzaquen, and E. Raphaël, Kelvin wake pattern at large Froude numbers, J. Fluid Mech. 738, R3 (2014). 
[24] D. E. Mowbray and B. S. H. Rarity, The internal wave pattern produced by a sphere moving vertically in a density stratified liquid, J. Fluid Mech. 30, 489 (1967).

[25] T. N. Stevenson, T. J. Woodhead, and D. Kanellopulos, Viscous effects in some internal waves, Appl. Sci. Res. 40, 185 (1983).

[26] U. Gärtner, U. Wernekinck, and W. Merzkirch, Velocity measurements in the field of an internal gravity wave by means of speckle photography, Exp. Fluids 4, 283 (1986).

[27] C. R. Torres, H. Hanazaki, J. Ochoa, J. Castillo, and M. Van Woert, Flow past a sphere moving vertically in a stratified diffusive fluid, J. Fluid Mech. 417, 211 (2000).

[28] S. Okino, S. Akiyama, and H. Hanazaki, Velocity distribution around a sphere descending in a linearly stratified fluid, J. Fluid Mech. 826, 759 (2017).

[29] See Supplemental Material at http://link.aps.org/supplemental/10.1103/PhysRevFluids.3.034801 for movies of the vertical velocity component of the wake for $(\operatorname{Re}=920, \operatorname{Ro}=0.65),(\operatorname{Re}=840, \operatorname{Ro}=1.97)$, and $(\operatorname{Re}=840, \operatorname{Ro}=19.7)$.

[30] C. H. K. Williamson, Vortex dynamics in the cylinder wake, Annu. Rev. Fluid Mech. 28, 477 (1996).

[31] E. Guyon, J.-P. Hulin, L. Petit, and C. D. Mitescu, Physical Hydrodynamics (Oxford University Press, Oxford, 2015).

[32] D. L. Boyer, P. A. Davies, H. J. S. Fernando, and X. Zhang, Linearly stratified flow past a horizontal circular cylinder, Philos. Trans. R. Soc. London Ser. A 328, 501 (1989).

[33] P. Meunier, Stratified wake of a tilted cylinder. Part 1. Suppression of a von Kármán vortex street, J. Fluid Mech. 699, 174 (2012).

[34] J. Boisson, D. Cébron, F. Moisy, and P.-P. Cortet, Earth rotation prevents exact solid-body rotation of fluids in the laboratory, Europhys. Lett. 98, 59002 (2012).

[35] H. K. Cheng, H. Hefazi, and S. N. Brown, Topographically generated cyclonic disturbance and lee waves in a stratified rotating fluid, J. Fluid Mech. 141, 431 (1984).

[36] T. M. J. Newley, H. J. Pearson, and J. C. R. Hunt, Stably stratified rotating flow through a group of obstacles, Geophys. Astrophys. Fluid Dyn. 58, 147 (1991).

[37] J. C. R. Hunt, Y. Feng, P. F. Linden, M. D. Greenslade, and S. D. Mobbs, Low-Froude-number stable flows past mountains, Il Nuovo Cimento 20C, 261 (1997).

[38] M. D. Greenslade, Drag on a sphere moving horizontally in a stratified fluid, J. Fluid Mech. 418, 339 (2000).

[39] J. C. R. Hunt, G. G. Vilenski, and E. R. Johnson, Stratified separated flow around a mountain with an inversion layer below the mountain top, J. Fluid Mech. 556, 105 (2006).

[40] S. B. Dalziel, M. D. Patterson, C. P. Caulfield, and S. Le Brun, The structure of low-Froude-number lee waves over an isolated obstacle, J. Fluid Mech. 689, 3 (2011).

[41] N. Bleistein, Mathematical Methods for Wave Phenomena (Academic, Orlando, 1984).

[42] N. Bleistein, Uniform asymptotic expansions of integrals with stationary point near algebraic singularity, Commun. Pure Appl. Math. 19, 353 (1966). 